RICE UNIVERISITY

\title{
Quantitative measurements of individual gold nanoparticle scattering cross sections
}

\author{
by
}

Lindsey J. E. Anderson

A THESIS SUBMITTED

IN PARTIAL FULFILLMENT OF THE

REQUIREMENTS FOR THE DEGREE

Master of Science

APPROVED, THESIS COMMITTEE:
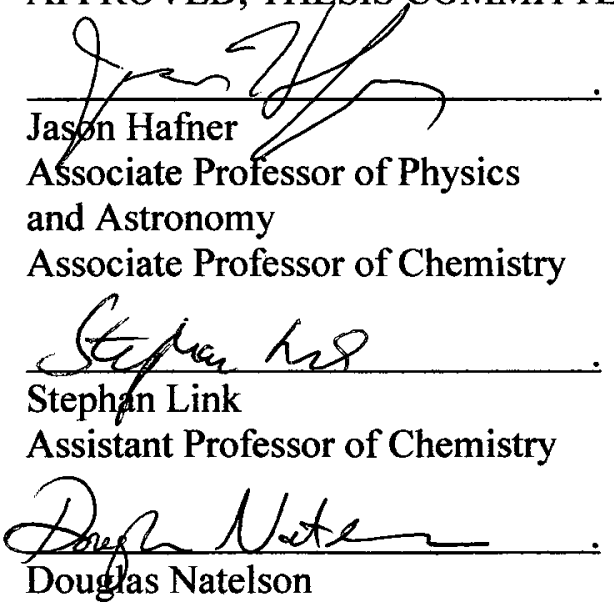

Associate Professor of Physics and Astronomy

Associate Professor of Electrical and Computer Engineering 
UMI Number: 1486058

All rights reserved

INFORMATION TO ALL USERS

The quality of this reproduction is dependent upon the quality of the copy submitted.

In the unlikely event that the author did not send a complete manuscript and there are missing pages, these will be noted. Also, if material had to be removed, a note will indicate the deletion.

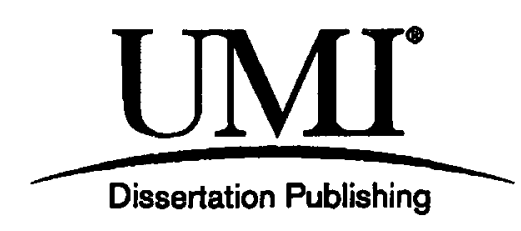

UMI 1486058

Copyright 2010 by ProQuest LLC.

All rights reserved. This edition of the work is protected against unauthorized copying under Title 17, United States Code.

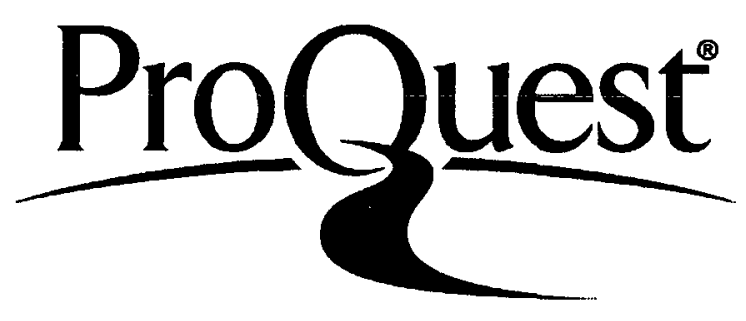

ProQuest LLC

789 East Eisenhower Parkway

P.O. Box 1346

Ann Arbor, Ml 48106-1346 


\title{
$\underline{\text { Abstract }}$
}

\section{Quantitative Measurements of Individual Gold Nanoparticle Scattering Cross Sections}

by

\author{
Lindsey Anderson
}

The local surface plasmon resonance (LSPR) of noble metal nanoparticles has recently been exploited in numerous applications. The LSPR peak position and linewidth have been studied quite extensively, but the magnitude of the resonance has not received much attention. Analytical solutions to Maxwell's Equations cannot predict the scattering cross section of arbitrarily-shaped particles at arbitrary illumination and detection angles. Dark field microscpectroscopy is a powerful tool for studying plasmon resonances of noble metal nanoparticles and for developing their applications in sensing and imaging.

We present a technique for calibrating dark field microspectrometer measurements to yield quantitative spectral scattering cross sections for arbitrarily shaped particles. Values for gold nanorods and gold bipyramids are reported. The measurements suggest that, for small elongated particles, the signal can be predicted by approximations based on the total cross section. 


\section{Acknowledgements}

I would like to thank my research group members for all of their help and support. Specifically, Kathryn Mayer and Colleen Nehl for assembling the single particle dark field microspecroscopy set up, Seunghyun Lee for his nanoparticle synthesis skills, Yi Yang for the preliminary research to get this project started, and Robbie Fraleigh for his assistance in sample fabrication. For helping me understand much of the underlying physics of plasmonics, I would like to thank Britian Willingham, Jorge Zuloaga and Kui Bao. I am lucky to have a wonderful advisor, Dr. Jason Hafner, who has always been extremely helpful and patient with me. I thank him for his ideas and the portion of his vast experimental knowledge which he has imparted to me. Lastly, I am appreciative of my family, who has always encouraged me to follow my passion for science. 


\section{Outline}

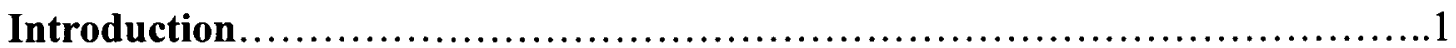

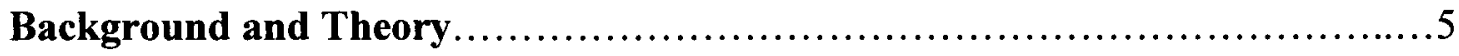

Scattering Cross Section of a Sphere.......................................5

Approximations and Extensions to the Mie Solution..........................

Dark-field Microscopy .................................................... 14

Methods..................................................................... 16

Sample Preparation.................................................... 16

Data Analysis........................................................ 17

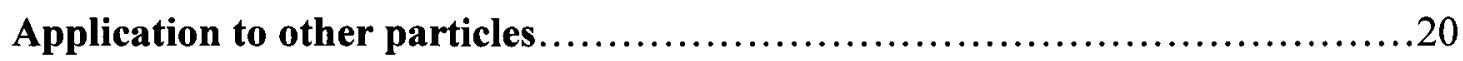

Gold Bipyramid......................................................20

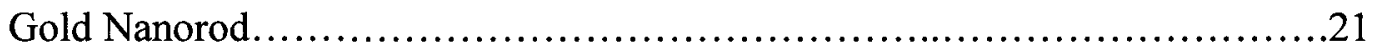

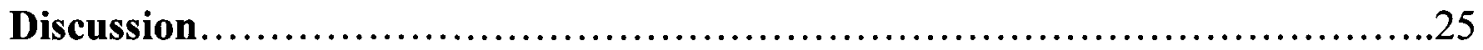

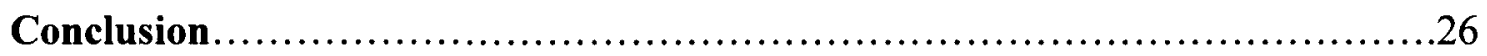

References............................................................ 27

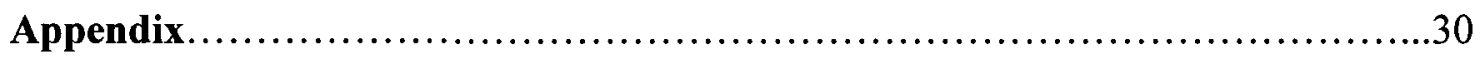




\section{Introduction}

Plasmons are defined as the classical oscillation of the free electron gas density in a metal. The oscillating field of electromagnetic radiation causes plasmon modes to propagate on the surface in a phenomenon known as surface plasmon resonance (SPR). Classically, the electrons can be thought of as an incompressible fluid and the SPR likened to a wave in this fluid. In this analogy, the incident light can be thought of as a "rock" hitting a "pond" of electrons. For metal particles on the order of or much smaller than the wavelength of incident light, the electrons collectively oscillate in what is termed a localized surface plasmon resonance (LSPR). On resonance, the extremely high confinement of the electromagnetic energy in and around the nanoparticle results in intense electric field enhancements. ${ }^{1}$ For these small particles, off resonant optical extinction cross section is smaller than the cross section of the particle itself due to diffraction (Figure 1A). On resonance, however, the extinction cross section can be larger than the physical cross section (Figure 1B). The dashed line in Figure 1B is the effective size of the particle when on resonance.
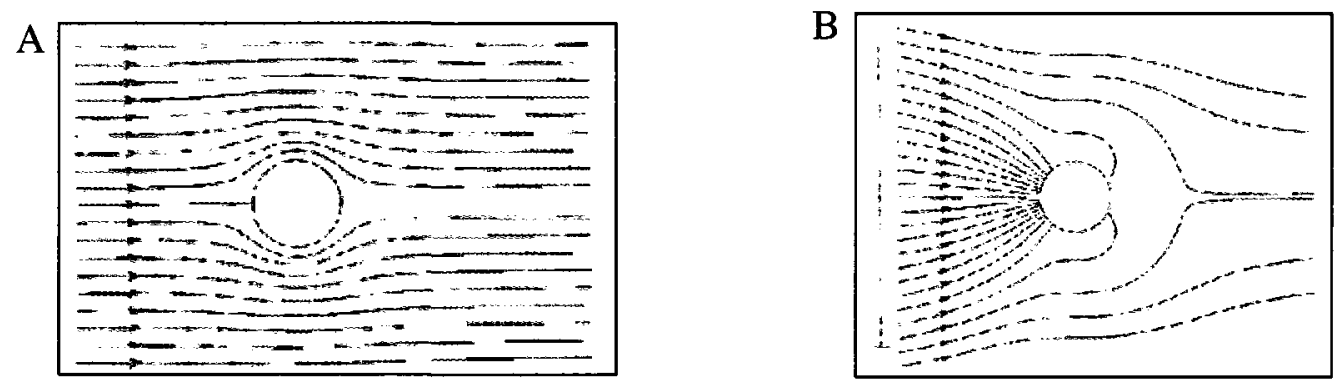

Figure 1 Field lines of the total Poynting vector (excluding the scattered) around a small aluminum sphere illuminated off resonance (A) and on resonance (B). The dashed line in B indicates the effective absorption radius of the sphere. ${ }^{2}$ 
The extinction cross section represents the total quantity of light removed from an incident plane wave. This occurs due to absorption and scattering, each of which have a corresponding cross section (Figure 2).

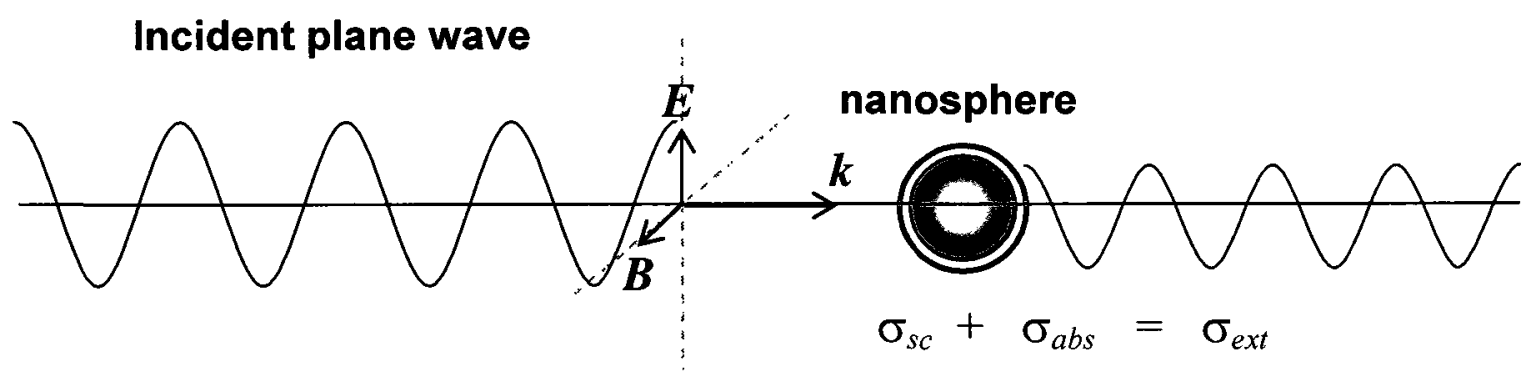

Figure 2 Schematic of an electromagnetic wave incident on a small gold sphere. Some of the incident radiation is absorbed by the particle, some is scattered, and the remainder continues traveling in the $k$ direction.

Noble metal nanoparticles and nanostructures exhibit LSPR at visible and nearinfrared frequencies. The resulting strong absorption, bright scattering, and enhanced near fields have generated great interest in these materials for applications in sensing, biomedical therapies and diagnostics, and nanophotonic circuits. ${ }^{3-6}$ Nanoparticle plasmon resonances can be characterized by a number of techniques, including ensemble spectral extinction measurements, ${ }^{7}$ single particle spectral scattering measurements, ${ }^{8-18}$ near field optical microscopy, ${ }^{19,} \quad 20$ electron energy loss spectroscopies, ${ }^{21,}{ }^{22}$ and cathodoluminsecence. $^{23,24}$ While these are well suited to measuring the resonant wavelength and spectral width, there are few reports on the precise experimental characterization of the amplitude of the resonance, which corresponds to the cross section. $^{25,26}$ Such characterization could be valuable for determining nanoparticle concentrations, predicting heat generation and scattering signals, testing the accuracy of numerical simulations, and possibly evaluating near field enhancements. ${ }^{27,} 28$ For 
intracellular imaging applications, particles need to be small enough to penetrate the cell wall, yet scatter a sufficient amount of detectable light. By quantifying the scattering cross section of different shaped nanoparticles, it will be possible to make particle choices that are application specific.

Single nanoparticle studies are particularly powerful for characterizing plasmon resonances since they avoid the confounding effect of nanoparticle heterogeneity. They are often carried out by dark field microspectroscopy at high numerical aperture $(>0.5)$, and have been applied to a variety of complex nanoparticle shapes for fundamental studies of the nature of the plasmon resonance. ${ }^{8-18}$ In their seminal paper, Schultz et al used single silver nanoparticles as specific biological labels for microscopic imaging, and estimated a single nanoparticle to provide approximately $10^{6}$ more signal than a fluorescein molecule and approximately $10^{5}$ more signal than a quantum $\operatorname{dot}^{29}$ The past decade has seen major advances in the synthesis of gold and silver nanoparticles with complex shapes and sizes that have also been considered for biological labeling. However, the measurements and estimates quoted above were for an $80 \mathrm{~nm}$ diameter silver nanoparticle, and the scattering signal from other plasmon resonant nanoparticles varies widely. The scattering cross section is strongly dependent on the nanoparticle size and shape, the peak resonance wavelength, the spectral variation of the metal dielectric properties, and the refractive index of the medium. In fact, the peak total scattering cross section for gold and silver nanospheres between 10 and $100 \mathrm{~nm}$ diameters varies over five orders of magnitude (Figure 3). 


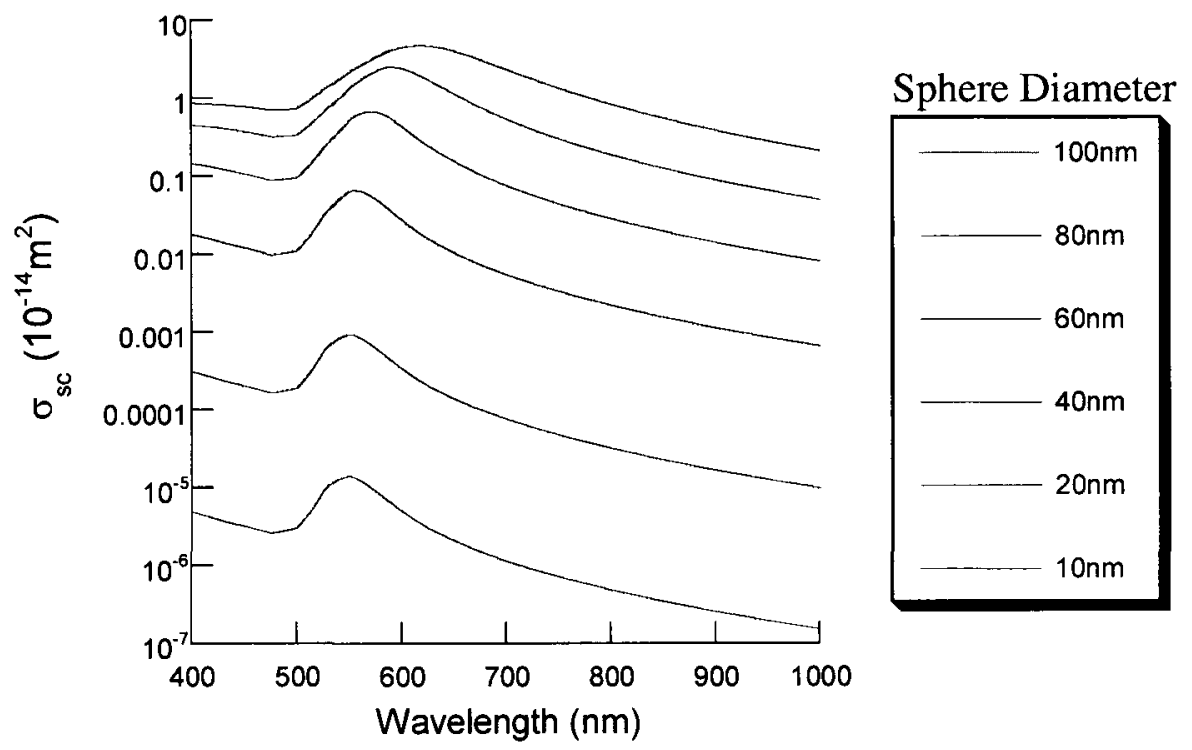

Figure 3 The total scattering cross section for gold nanospheres in a medium of refractive index 1.515 .

The scattering signal collected in imaging applications will depend on the illumination angle and differential scattering cross section integrated over the solid angle of the imaging lens, so predictions based on the total scattering cross section can be misleading. While there have been some numerical simulations of the effect of nanoparticle size and shape on the magnitude of the scattering cross section, ${ }^{30-32}$ the lack of direct experimental comparison has left plasmon resonant nanoparticles' scattering properties ambiguous and at times perhaps even overstated. We present an effort to quantify the scattering from plasmon resonant nanoparticles with complex shapes by dark field microspectroscopy. 


\section{Background and Theory}

\section{Scattering Cross Section of a Sphere}

The problem of scattering from a sphere due to an incident plane wave can be solved analytically. Following Bohren and Huffman, ${ }^{2}$ a realizable time-harmonic electromagnetic field in a linear, isotropic, homogeneous medium must satisfy the wave equation:

$$
\nabla^{2} \mathbf{E}+k^{2} \mathbf{E}=\mathbf{0} \quad \nabla^{2} \mathbf{H}+k^{2} \mathbf{H}=\mathbf{0}
$$

where $\mathrm{k}^{2}=\omega^{2} \varepsilon \mu$, and have no divergence:

$$
\nabla \cdot \mathbf{E}=\mathbf{0} \quad \nabla \cdot \mathbf{H}=\mathbf{0}
$$

Additionally, $\mathbf{E}$ and $\mathbf{H}$ are not independent:

$$
\nabla \times \mathbf{E}=i \omega \mu \mathbf{H} \quad \nabla \times \mathbf{H}=-i \omega \varepsilon \mathbf{E}
$$

We can construct vector functions which satisfy these requirements:

$$
\mathbf{M}=\nabla \times(\mathbf{c} \varphi) \quad \mathbf{N}=\frac{\nabla \times \mathbf{M}}{k}
$$

where $\varphi$ is a scalar function and $\mathbf{c}$ is an arbitrary constant. $\mathbf{M}$ and $\mathbf{N}$ have all of the required properties of an electromagnetic field: they satisfy the vector wave equation, they are divergence free, and the curl of one is proportional to the other. Furthermore, it can be shown that if $\mathbf{M}$ satisfies the vector wave equation, $\varphi$ satisfies the scalar wave equation. Therefore, the problem of finding solutions to the field equations reduces to the simpler problem of finding solutions to the scalar wave equation (for $\varphi$ ). $\varphi$ is termed the generating function for the vector harmonics $\mathbf{M}$ and $\mathbf{N}$ and $\mathbf{c}$ is called the guiding or pilot 
vector. Due to the spherical symmetry of the problem, we choose functions $\varphi$ that satisfy the wave equation in spherical coordinates and $\mathbf{c}$ to be the radius vector $\mathbf{r}$.

In spherical coordinates, $\varphi$ is of the form:

$$
\varphi(r, \theta, \phi)=R(r) \Theta(\theta) \Phi(\phi)
$$

Inserting this into the wave equation in spherical coordinates, we have 3 separated equations:

$$
\begin{gathered}
\frac{d^{2} \Phi}{d \phi^{2}}+m^{2} \Phi=0 \\
\frac{1}{\sin \theta} \frac{d}{d \theta}\left(\sin \theta \frac{d \Theta}{d \theta}\right)+\left[n(n+1)-\frac{m^{2}}{\sin ^{2} \theta}\right] \Theta=0 \\
\frac{d}{d r}\left(r^{2} \frac{d R}{d r}\right)+\left[k^{2} r^{2}-n(n+1)\right] R=0
\end{gathered}
$$

where $\mathrm{m}$ and $\mathrm{n}$ are integers. For $\Phi$, the linearly independent solutions are $\Phi_{e}=\cos m \phi$ and $\Phi_{o}=\sin m \phi$. Solutions to (2) are the associated Legendre functions of the first kind $P_{n}^{m}(\cos \theta)$ of degree $n$ and order $m(n=m, m+1, \ldots)$. The Legendre functions ultimately give the angular dependence of the scattering cross section. Finally, the linearly independent solutions to (3) are the spherical Bessel functions or the first and second kind. By plugging these solutions into $\mathbf{M}$ and $\mathbf{N}$, we get $\mathbf{M}_{\mathrm{emn}}, \mathbf{M}_{\mathrm{omn}}, \mathbf{N}_{\mathrm{emn}}$ and $\mathbf{N}_{\text {omn }}$. The scattering fields can be expressed as linear combinations of these vector spherical harmonics. Due to orthogonality, only Legendre functions of the first order are nonzero.

The incident plane wave is expressed in spherical coordinates as

$$
\mathbf{E}_{i}=\mathbf{E}_{0} \sum_{n=1}^{\infty} i^{n} \frac{2 n+1}{n(n+1)}\left(\mathbf{M}_{o 1 n}^{(1)}-i \mathbf{N}_{e 1 n}^{(1)}\right) \quad \mathbf{H}_{i}=\frac{-k}{\omega \mu} E_{0} \sum_{n=1}^{\infty} i^{n} \frac{2 n+1}{n(n+1)}\left(\mathbf{M}_{e 1 n}^{(1)}-i \mathbf{N}_{o 1 n}^{(1)}\right)
$$


After imposing particle boundary conditions and the orthogonality of the vector harmonics, we arrive at an expression for the scattered fields.

$$
\mathbf{E}_{s}=\sum_{n=1}^{\infty} E_{n}\left(i a_{n} \mathbf{N}_{e 1 n}^{(3)}-b_{n} \mathbf{M}_{o 1 n}^{(3)}\right) \quad \mathbf{H}_{s}=\frac{k}{\omega \mu} \sum_{n=1}^{\infty} E_{n}\left(i a_{n} \mathbf{N}_{o 1 n}^{(3)}-b_{n} \mathbf{M}_{e 1 n}^{(3)}\right)
$$

where

$$
a_{n}=\frac{m \psi_{n}(m x) \psi_{n}^{\prime}(x)-\psi_{n}(x) \psi_{n}^{\prime}(m x)}{m \psi_{n}(m x) \xi_{n}^{\prime}(x)-\xi_{n}(x) \psi_{n}^{\prime}(m x)} \quad b_{n}=\frac{\psi_{n}(m x) \psi_{n}^{\prime}(x)-m \psi_{n}(x) \psi_{n}^{\prime}(m x)}{\psi_{n}(m x) \xi_{n}^{\prime}(x)-m \xi_{n}(x) \psi_{n}^{\prime}(m x)}
$$

$m$ is the relative refractive index $\left(m=n_{p} / n_{m}\right), n_{p}$ is the complex refractive index of the nanoparticle material, $n_{m}$ is the refractive index of the medium, $x=k r$ where $r$ is the nanoparticle radius, and $\psi_{n}$ and $\xi_{n}$ are the Riccati-Bessel functions. The incident and scattered fields in each polarization state can be related by the amplitude scattering matrix as follows:

$$
\left(\begin{array}{l}
E_{/ / s} \\
E_{\perp s}
\end{array}\right)=\frac{e^{i k(r-z)}}{-i k r}\left(\begin{array}{cc}
S_{2} & S_{3} \\
S_{4} & S_{1}
\end{array}\right)\left(\begin{array}{l}
E_{/ / i} \\
E_{\perp i}
\end{array}\right)
$$

where the incident plane wave is traveling in the $\mathrm{z}$-direction. Writing this equation in a different way, it is possible to relate the intensity and polarization of the incident and scattered waves.

$$
\left(\begin{array}{l}
I_{s} \\
Q_{s} \\
U_{s} \\
V_{s}
\end{array}\right)=\frac{1}{k^{2} r^{2}}\left(\begin{array}{llll}
S_{11} & S_{12} & S_{13} & S_{14} \\
S_{21} & S_{22} & S_{23} & S_{24} \\
S_{31} & S_{32} & S_{33} & S_{34} \\
S_{41} & S_{42} & S_{43} & S_{44}
\end{array}\right)\left(\begin{array}{c}
I_{i} \\
Q_{i} \\
U_{i} \\
V_{i}
\end{array}\right)
$$

Where

$$
I_{s}=\left\langle E_{/ / s} E_{/ / s}^{*}+E_{\perp s} E_{\perp s}^{*}\right\rangle, Q_{s}=\left\langle E_{/ / s} E_{/ / s}^{*}-E_{\perp s} E_{\perp s}^{*}\right\rangle, U_{s}=\left\langle E_{/ / s} E_{\perp s}^{*}+E_{\perp s} E_{/ l s}^{*}\right\rangle, V_{s}=i\left\langle E_{/ l s} E_{\perp s}^{*}-E_{\perp s} E_{/ l s}^{*}\right\rangle
$$

The elements of the scattering matrix are known as the Stokes parameters. Our experiments are done with unpolarized light, for which the state vector is $\left(\begin{array}{llll}1 & 0 & 0 & 0\end{array}\right)$, so we 
are only interested in the Stokes parameter $S_{11}=\frac{I_{s}}{I_{i}}=\frac{1}{2}\left(\left|S_{1}\right|^{2}+\left|S_{2}\right|^{2}\right)$, where $I$ is simply the irradiance. Here we are interested in differential cross sections, which are experimentally relevant: $\frac{d \sigma_{s c}}{d \Omega}=r^{2} S_{11}$. The scattering parameters $S_{1}$ and $S_{2}$ incorporate the angular functions defined as $\pi_{n}=P_{n}^{I} / \sin \theta$ and $\tau_{n}=d P_{n}^{l} / d \theta$ where $\theta$ is the polar angle of the scattered light relative to the incoming wave vector,

$$
S_{1}=\sum_{n} \frac{2 n+1}{n(n+1)}\left(a_{n} \tau_{n}+b_{n} \pi_{n}\right) \quad S_{2}=\sum_{n} \frac{2 n+1}{n(n+1)}\left(a_{n} \pi_{n}+b_{n} \tau_{n}\right)
$$

Using these expressions, we arrive at the differential scattering cross section:

$$
\frac{d \sigma_{s c}}{d \Omega}=\frac{1}{2 k_{m}^{2}}\left(\left|\sum_{n} \frac{2 n+1}{n(n+1)}\left(a_{n} \pi_{n}+b_{n} \tau_{n}\right)\right|^{2}+\left|\sum_{n} \frac{2 n+1}{n(n+1)}\left(a_{n} \tau_{n}+b_{n} \pi_{n}\right)\right|^{2}\right)
$$

where $k_{m}$ is the wavenumber of the light in the surrounding medium $\left(k_{m}=2 \pi n_{m} / \lambda\right)$, and $\lambda$ is the vacuum wavelength. The sums over $n$ are over all multipole excitations. The total scattering cross section is expressed as:

$$
\sigma_{s c}=\frac{2 \pi}{k^{2}} \sum_{n=1}^{\infty}(2 n+1)\left(\left|a_{n}\right|^{2}+\left|b_{n}\right|^{2}\right)
$$

A MATLAB program was written to calculate the differential and total scattering cross sections of spherical particles of a chosen material in a defined dielectric medium (Appendix A). Polar plots of the angular scattered irradiance for a water droplet in air show the expected dipole for small particles (Figure 4) for light polarized parallel (red) and perpendicular (green) to the scattering plane. As particle size increases, however, a larger fraction of the total scattering is in the same direction as the incident light (forward scattering). With the incoming light is along $0^{\circ}$, the plots in Figure 5 show the angular scattering pattern for increasing water droplet size. The black plots (left) are taken 
directly from Bohren \& Huffman and the colored plots (right) were made to verify the calculations. These plots illustrate the importance of accounting for the size and angle of the objective when comparing dark field measurements with calculations, i.e. it is not safe to assume that the same fraction of scattered light is being measured for different particles. Furthermore, the scattering patterns are most likely much more complex for nonspherical particles.

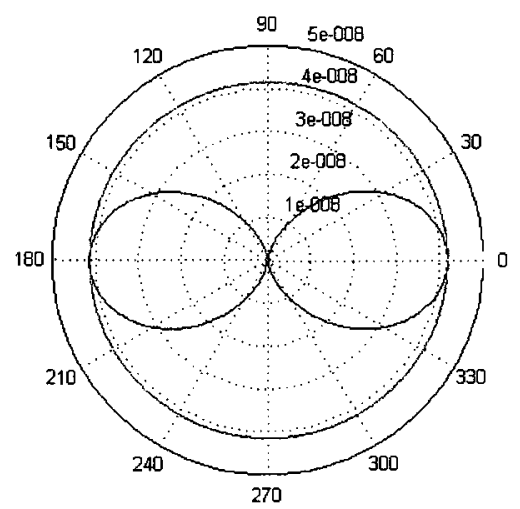

Figure 4 Dipolar scattering pattern for a spherical water droplet in the small particle limit for light polarized parallel (red) and perpendicular (green) to the scattering plane.

\section{Approximations and Extensions to the Mie Solution}

The total cross section expressions from the Mie solution can be simplified by assuming particles that are much smaller than the wavelength of the incident light. Sometimes referred to as "the dipole approximation", the small particle approximation not only omits all but the $n=1$ term in Equation 5 (dipole term), but also assumes that ( $k r$ $<<1$ ). In this case, only the first few terms in the power series expansions of the spherical Bessel functions are significant. The Mie solution for the total scattering cross section reduces to:

$$
\sigma_{s c}=\frac{24 \pi^{3} V^{2} \varepsilon_{m}^{2}}{\lambda^{4}}\left[\frac{\left(\varepsilon_{1}-\varepsilon_{m}\right)^{2}+\varepsilon_{2}^{2}}{\left(\varepsilon_{1}+2 \varepsilon_{m}\right)^{2}+\varepsilon_{2}^{2}}\right]
$$



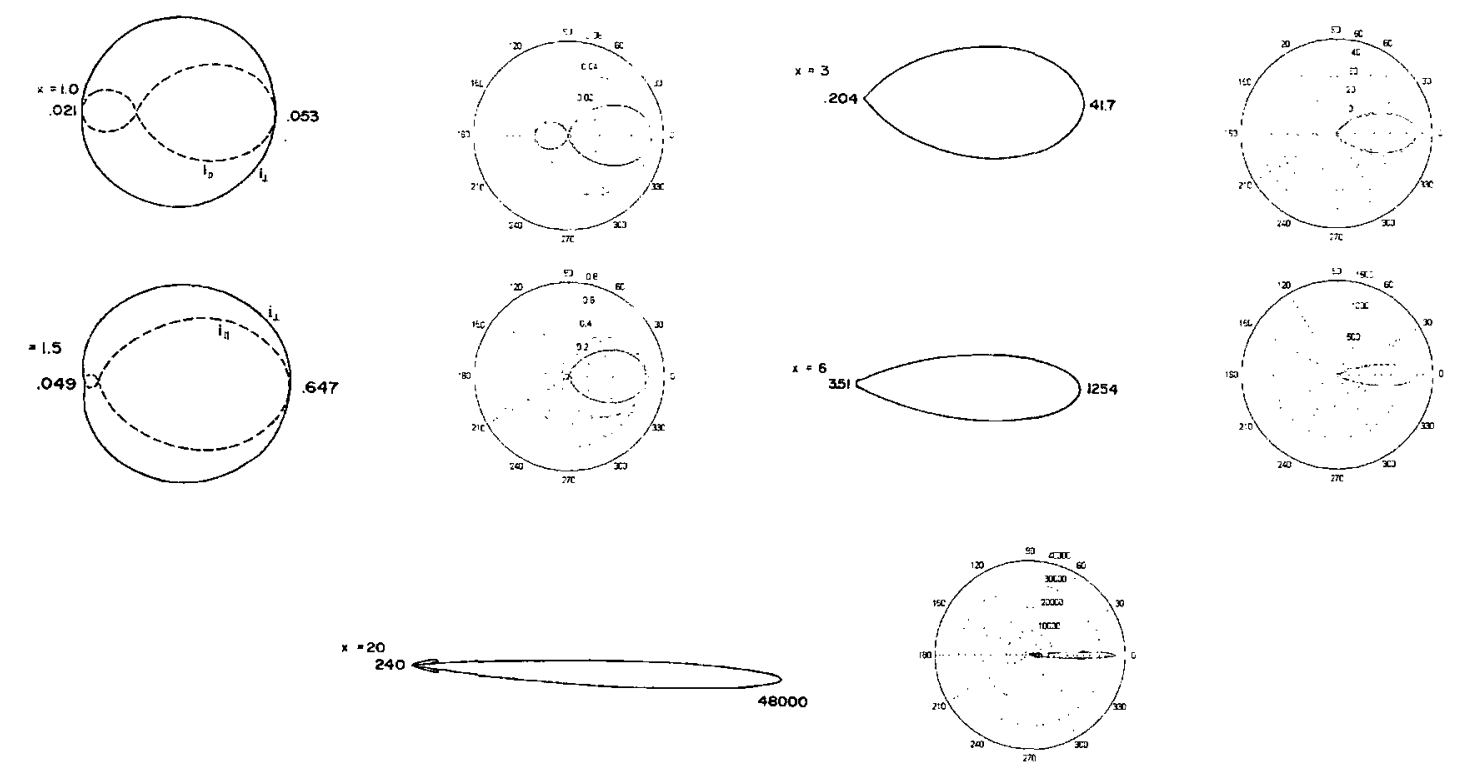

Figure 5 Angular scattering irradiance of a spherical water droplet where $x=2 \pi n_{p} r / \lambda$. Forward scattering increases as the ratio of the particle size increases. Parallel (dashed and red lines) and perpendicular (solid and green lines) polarization of incident light.

where $V$ is the nanoparticle volume, $\varepsilon_{m}$ is the dielectric function of the medium, and $\varepsilon_{1}+$ $i \varepsilon_{2}$ is the complex dielectric function of the nanoparticle. Note that complex refractive indices are often used to express the full Mie solution, but approximations for small particles are most succinctly written with the corresponding dielectric functions.

The Mie solution can be extended to spheroids in this small particle limit, yielding the following expression, known as the Gans Solution:

$$
\sigma_{s c}=\frac{8 \pi^{3} V^{2} \varepsilon_{m}^{2}}{9 \lambda^{4}} \sum_{j} \frac{\frac{1}{P_{j}^{2}}\left[\left(\varepsilon_{1}-\varepsilon_{m}\right)^{2}+\varepsilon_{2}^{2}\right]}{\left(\varepsilon_{1}+\frac{1-P_{j}}{P_{j}} \varepsilon_{m}\right)^{2}+\varepsilon_{2}^{2}}
$$

where $P_{j}$ are depolarization factors which depend on the nanoparticle aspect ratio. ${ }^{33}$

$$
P_{A}=\frac{1-e^{2}}{e^{2}}\left[\frac{1}{2 e} \ln \frac{1+e}{1-e}-1\right] \quad P_{B}=P_{C}=\frac{1-P_{A}}{2}
$$


For prolate or elongated ellipsoids $(\mathrm{A}>\mathrm{B}=\mathrm{C})$ where $e=\left(\frac{A^{2}-B^{2}}{A^{2}}\right)^{1 / 2}$. To gauge the size range over which such approximations are accurate, Figure 6 displays the total scattering cross section calculated by Equation 5 (full Mie solution) and Equation 6 (small particle approximation) for 10,60 , and $100 \mathrm{~nm}$ diameter gold nanospheres. The MATLAB program in Appendix B was used for all calculations in this section. The small particle approximation is quite accurate for the $10 \mathrm{~nm}$ diameter nanosphere, it is blueshifted but of similar magnitude for the $60 \mathrm{~nm}$ sphere, and it is completely inaccurate for the $100 \mathrm{~nm}$ sphere
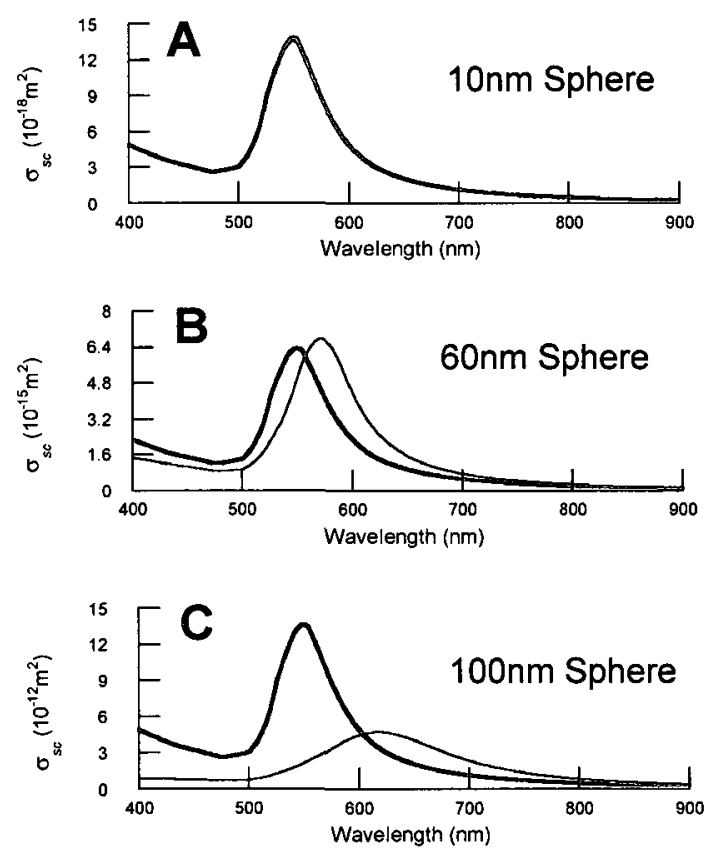

Figure 6 The total scattering cross sections for gold nanospheres calculated by the full Mie solution (blue) and the small particle approximation (orange) for $10 \mathrm{~nm}(\mathrm{~A}), 60 \mathrm{~nm}(\mathrm{~B})$, and $100 \mathrm{~nm}$ (C) diameter gold nanospheres.

It should be noted that referring to Equation 6 or a similar expression for the extinction as "the dipole approximation" is a bit of a misnomer. If we calculate the scattering cross section using only the dipole term (Equation $4, n=1$ ), the calculations agree with the full 
Mie expression (summing over all multipole terms) for spheres larger than 100nm (Figure 7). It is the small particle approximation which simplifies the expression, and which makes the solution valid only for particles below $100 \mathrm{~nm}$ diameter.
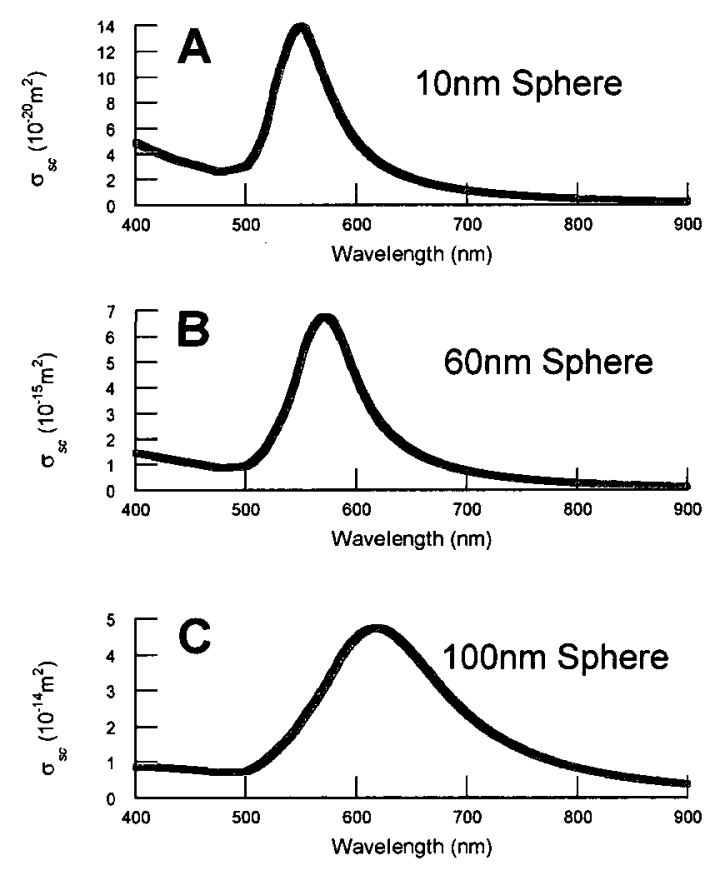

Figure 7 Scattering cross sections for gold nanospheres of diameter $10 \mathrm{~nm}, 60 \mathrm{~nm}$, and $100 \mathrm{~nm}$ in oil. The dipole term only (green) agrees well with the full Mie expression (red).

Full electromagnetic solutions for scattering from spheroids which could yield the differential scattering cross section for elongated nanoparticles have been derived, ${ }^{34}$ but they have not yet been applied to nanoparticle plasmon resonances. Numerical simulations are certainly the most straightforward approach to predicting the brightness of a specific nanoparticle of arbitrary shape. Several numerical methods have been employed to calculate the scattering properties of non-spherical particles, including Finite Element Method, Boundary Element Method, Finite Difference Time Domian, T-Matrix and Discrete Dipole Approximation, but they typically report the total cross section, ${ }^{30-32,}$ 35,36 so they cannot be directly compared to the results presented here. Recently, 
numerical simulations have been reported which exactly match dark field measurements of nanoparticles on substrates. ${ }^{37}$ If such simulations were designed to also report scattering cross sections, they could be quantitatively compared to the experimental observations described here.

As shown above, the Mie solution including angular dependence is the only analytical solution applicable to the problem of single particle scattering in the dark-field geometry for particles in the $50-100 \mathrm{~nm}$ range. Therefore, this solution can be used to calibrate dark-field measurements with spheres to obtain the scaling constant between the scattering cross section at the angle of the objective and the CCD counts per second. After finding this constant for a specific microscope system, the constant can be used to find the scattering cross section at the angle of the objective of arbitrarily shaped particles. 


\section{Dark-field Microscopy}

The epi-illuminated dark field imaging geometry is illustrated in Figure 8. The illumination is directed to the sample from an annular ring around the objective lens. For an optically flat substrate, only light scattered from the nanoparticle enters the objective while the remainder of the light passes through the glass. If one assumes constant incident irradiance $E_{i n c}$, to the nanoparticle, then the scattered radiant power that enters the objective, $\Phi_{s c a}$, will be proportional to the nanoparticle differential scattering cross section integrated over the solid area of the objective lens.

A

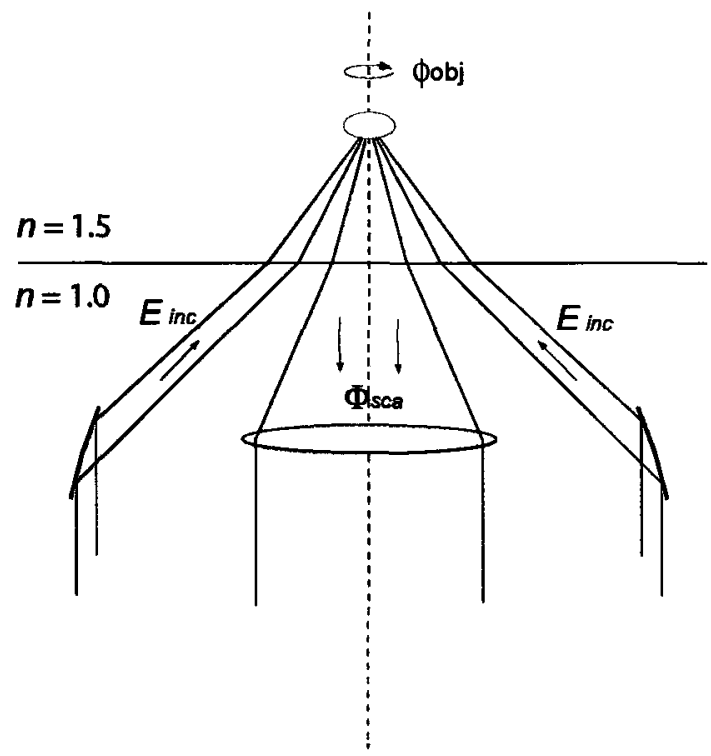

B

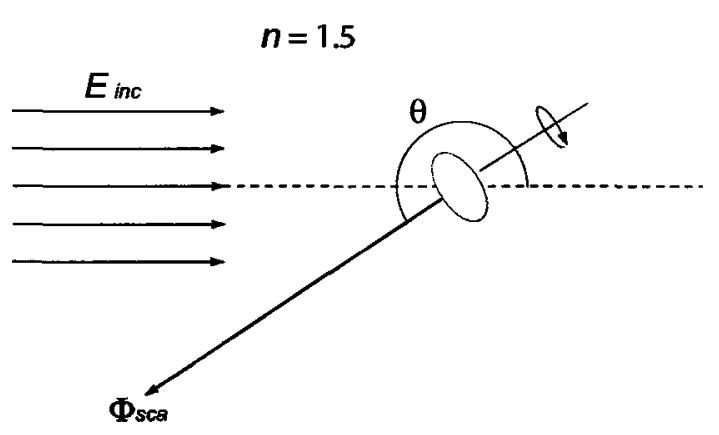

Figure 8 Schematics of the dark field imaging and scattering geometries. A. Annular illumination is directed toward a nanoparticle under oil immersion, and the scattering enters the objective lens along the optical axis of the microscope. B. The equivalent scattering diagram shows that the dark field measurements provide a measure of the backscattered differential scattering cross section at an angle of 207 degrees from the illumination axis, integrated over the aperture of the objective. The resulting scattering signal is an azimuthal average around the direction of the microscope optical axis as illustrated. 
We refer to this integrated value of the differential cross section as $\sigma_{o b j}$. When the scattered light reaches the CCD, it will generate a count rate that is proportional to $\Phi_{s c a}$, and therefore proportional to $\sigma_{o b j}$. For the dark field microscope objective used here (Zeiss LD Epi-plan 50x/0.5), the angle between the annular illumination and the optical axis of the objective lens is 45 degrees, and the numerical aperture of the objective indicates that light is collected into a 30 degree cone. The corresponding scattering diagram is shown in Figure 8B. If the optical axis is now taken to be along the incident illumination direction, one sees that the differential scattering cross section measured is backscattering approximately 225 degrees from the incident direction.

To achieve experimental conditions that match Mie scattering theory (see Figure 8A), nanoparticles were deposited onto glass substrates and covered with index matching oil and a cover slip so that the nanoparticles were in a homogenous dielectric medium rather than at a dielectric air-glass interface. The objective lens is not designed for oil immersion, but since it has a long working distance $(6.7 \mathrm{~mm})$, it images well through the thin cover slip and oil layer $(0.2 \mathrm{~mm})$. The air-glass dielectric interface, although now separated from the nanoparticle, will affect the incidence and scattering angles. By approximating the imaging system with geometrical optics, the scattering angle $\theta$ was calculated to be 207 degrees. Since the illumination and light collection occur around the optical axis of the objective, the CCD signal is proportional to the azimuthally-averaged differential section around $\sigma_{o b j}$, as illustrated in Figure 8B. For a nanoparticle with azimuthal symmetry (such as a nanosphere), this azimuthal dependence can be ignored. 


\section{Methods}

\section{Sample preparation}

Gold nanospheres (Ted Pella) were deposited sparsely on a glass substrate (Figure 9A). In order to correctly characterize each particle the location needed to be verified. An indexed TEM grid was placed on top of the slide then chrome and gold were evaporated onto the slide. After removal of the TEM grid, bare glass with particles is left in the covered areas (Figure 9B). Individual, isolated gold nanospheres were found and confirmed by correlated atomic force microscopy (AFM) and dark field imaging. Before measuring the single particle spectrum, index-matched oil was dropped on the slide, covered with a cover slip and sealed (Figure 9C).

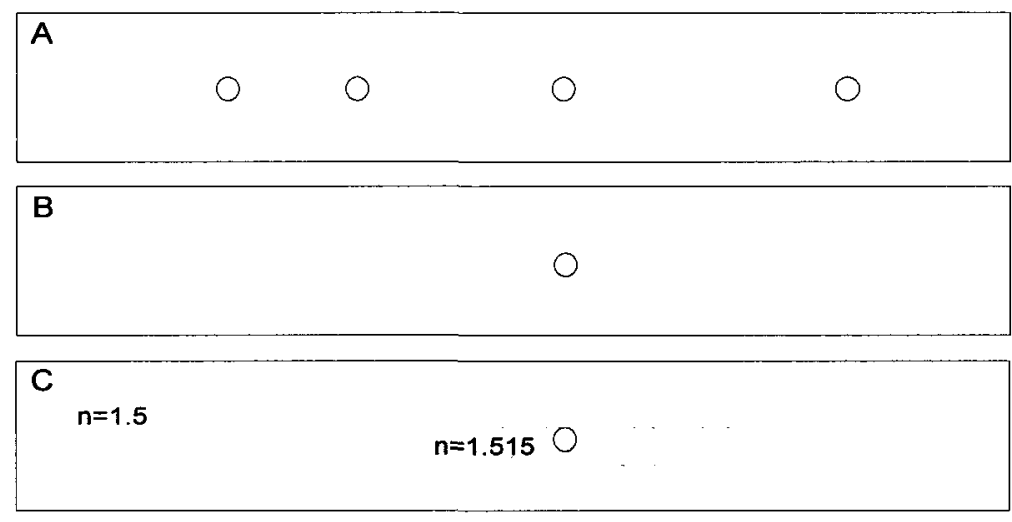

Figure 9 Schematic of nanoparticles face-down on a glass substrate (the dark-field illumination and detection below the substrate) (A). An index TEM grid is placed on the substrate (particle side) and a gold film is evaporated onto the surface, leaving some particles on bare glass after the removal of the grid (B). The particles are immersed in oil (grey) and covered to create an effectively continuous dielectric medium (C).

Following oil immersion, the spectrum of individual particles was measured with the dark-field microspectrometer. Figure 10 shows the dark-field image of a single particle on glass within the indexed TEM grid. The particles were isolated in a horizontal 
slit, then the mirror reflecting light into the CCD was changed to a diffraction grating in order to measure the particle spectrum.

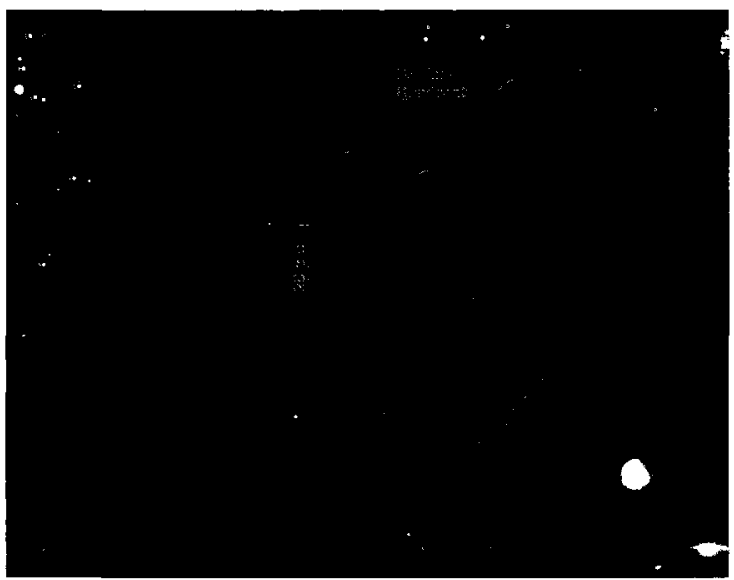

Figure 10 A dark field image of the particle substrate with the index grid. The green point is a single gold nanoparticle.

\section{$\underline{\text { Data analysis }}$}

An example of a CCD image using the diffraction grating is shown in Figure 11. The bright line near the center of the image is the image of a single gold nanospheres dispersed in wavelength along in the vertical direction. The particle is isolated in a horizontal slit. The vertical axis is the wavelength of the scattered light at that particular position along the slit. The bright objects near the edges are due to the scattering of the alignment marks. 


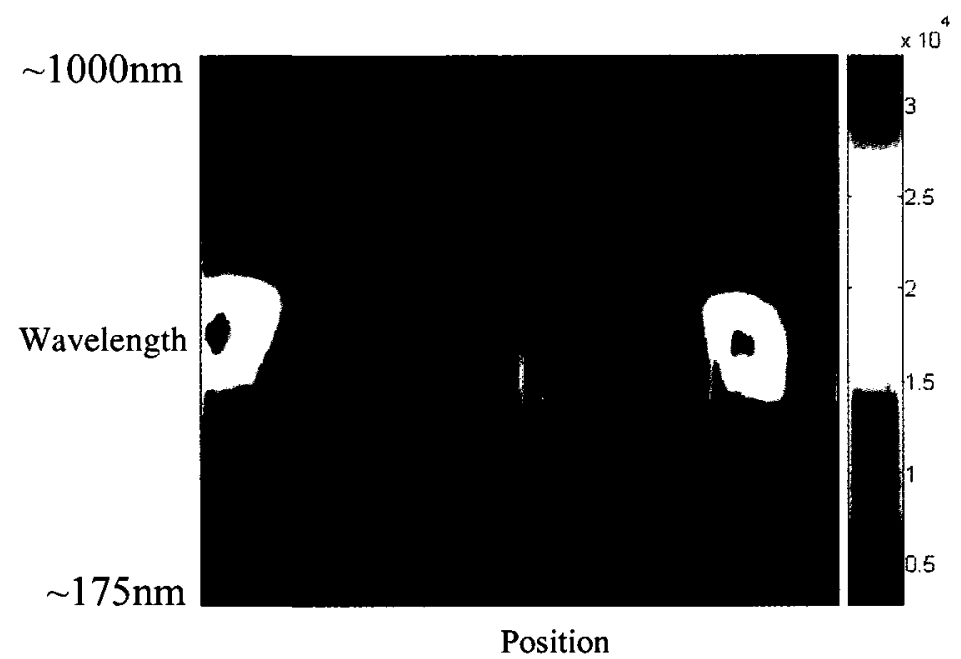

Figure 11 CCD image. The horizontal axis is position and the vertical axis is the wavelength. The color intensity reveals the scattering intensity at the respective wavelength and position.

These images were processed in MATLAB (Appendix C) in order to obtain a spectrum. Based on the image, the data analysis program asks the user to specify the particle position and width in pixels over which it sums the intensity. The background near the particle is fit to a polynomial of an order specified by the user and is subtracted from the particle signal. The result after the user enters the integration time of the image is the CCD counts per second as a function of the wavelength. Figure 12A displays the dark field spectral scattering rate for nanospheres that are $79 \mathrm{~nm}$ and $109 \mathrm{~nm}$ in diameter with the AFM images inset. For calibration, the differential scattering cross sections of the spheres were calculated from Mie scattering theory. The calculations included the first ten terms, and were integrated over the solid angle of the microscope objective to find $\sigma_{o b j}$. The resulting cross sections are plotted in Figure 12B. The relative spectral magnitudes of the count rates and Mie calculations match quite well. Figure 12C displays the spectral "scattering constant" for the dark field microspectrometer, $K_{\square}$, found by dividing the count rate by $\sigma_{o b j}$ at each wavelength. Error bars, determined from the experimental CCD counts in Figure $12 \mathrm{~A}$, are included. Since $K_{\lambda}$ is simply a calibrated 
spectral efficiency of the dark field microspectrometer and independent of the sample, the 79 and $109 \mathrm{~nm}$ diameter nanoparticles yield similar curves (Figure 12C). $K_{\lambda}$ can therefore be used to convert CCD count rates to cross sections for other nanoparticle shapes. Measurements on nanorods and bipyramids were analyzed with an average of the two curves presented in Figure 12C.
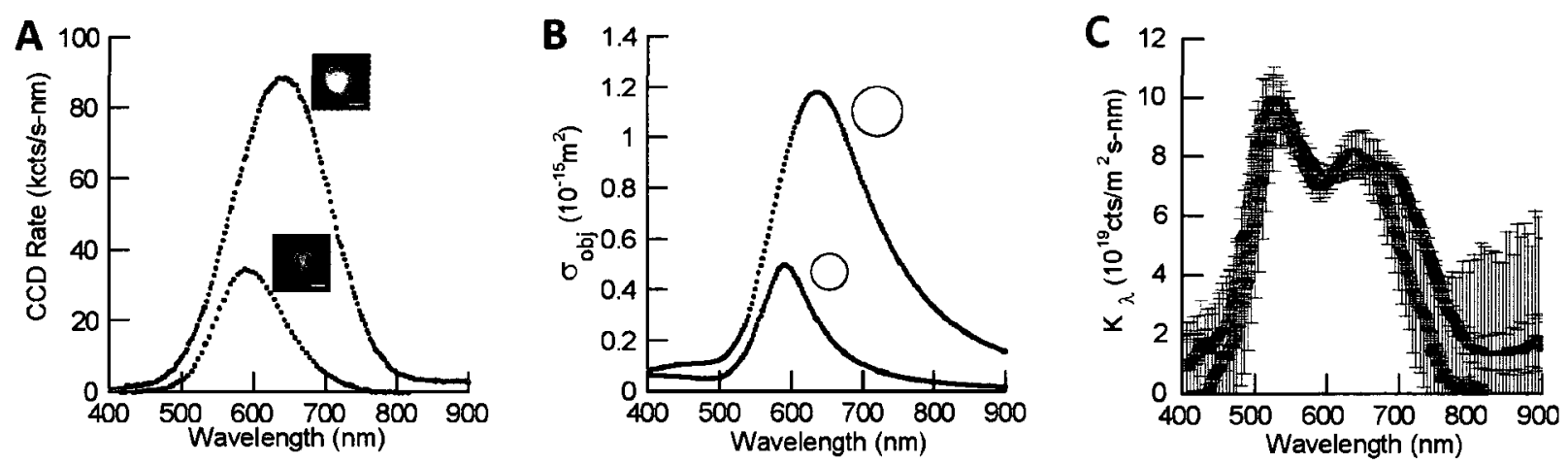

Figure 12 Calibration of the dark field scattering signal. A. The spectral CCD count rate from two nanospheres of $79 \mathrm{~nm}$ and $109 \mathrm{~nm}$ diameter with inset AFM images (scale bar is $50 \mathrm{~nm}$ ). B. The calculated differential cross sections of the same nanospheres integrated over the solid angle of the objective. $C$. The resulting spectral scattering constant. 


\section{Application to other particles}

\section{Gold bipyramid}

Gold bipyramids have the shape of two elongated pentagonal pyramids connected at their bases. Bipyramids are synthesized by surfactant-directed growth (as are gold nanorods), but are much more monodisperse and generally larger in size. ${ }^{18}$ An individual $135 \times 53 \mathrm{~nm}$ bipyramid was located relative to alignment marks and characterized by environmental scanning electron microscopy (ESEM). The bipyramid was then immersed in oil, located in the dark field microspectrometer and its spectral count rate recorded. The spectral scattering constant was applied to convert the data to the $\sigma_{o b j}$ presented in Figure 13. As mentioned above, $\sigma_{o b j}$ represents the azimuthal average of the bipyramid differential cross section integrated over the objective lens's solid angle. The peak scattering cross section to the objective is $1.2 \times 10^{-16} \mathrm{~m}^{2}$, and the narrow spectral width is consistent with previous reports of single bipyramid spectra. ${ }^{18}$

In addition to the cross section, Figure 13 displays $\sigma_{o b j}$ for a sphere of the equivalent volume calculated from the full Mie solution as in Figure 12. Under the imaging conditions reported here, the bipyramid is approximately two times brighter than its equivalent sphere. 


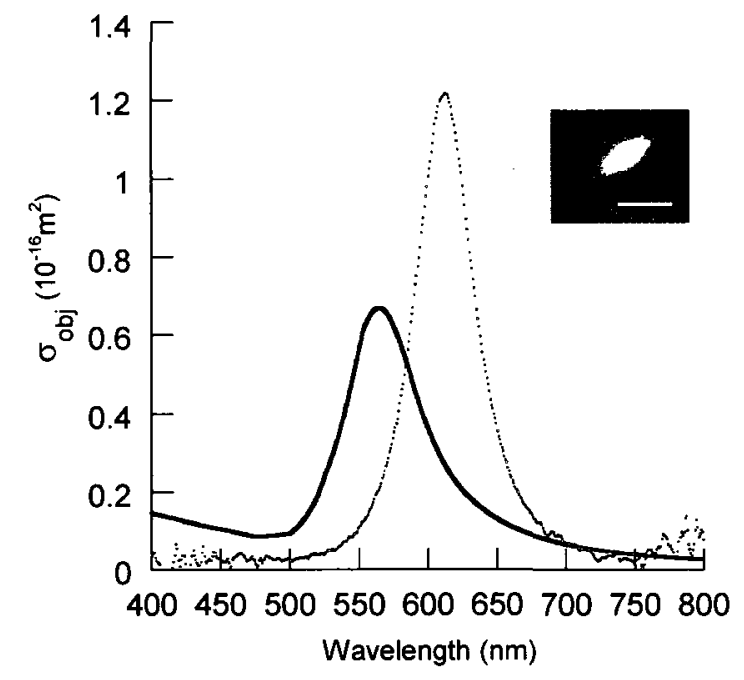

Figure 13 The measured gold bipyramid scattering cross section (red) and the calculated cross section for a gold sphere of equivalent volume (green). The inset shows an ESEM image of the bipyramid on glass (scale bar is $100 \mathrm{~nm}$ ).

\section{Gold nanorod}

In an attempt to measure single nanorod scattering, we have found a that nanorods (up to $50 \times 15 \mathrm{~nm}$ ) do not scatter sufficiently to be detected by dark field microscopy at $\mathrm{NA}=0.5$ with a halogen lamp illumination. ${ }^{38}$ Figure 14 shows AFM images (A and B) of a single gold nanorod. Using two detectors, a high sensitivity $\mathrm{CCD}(\mathrm{C})$ and a color $\mathrm{CCD}$ (D), the individual particle does not scatter a detectable amount of light. These small, dim particles, however, can be imaged by more sensitive methods such as confocal reflectance microscopy. ${ }^{35,39,40}$ 


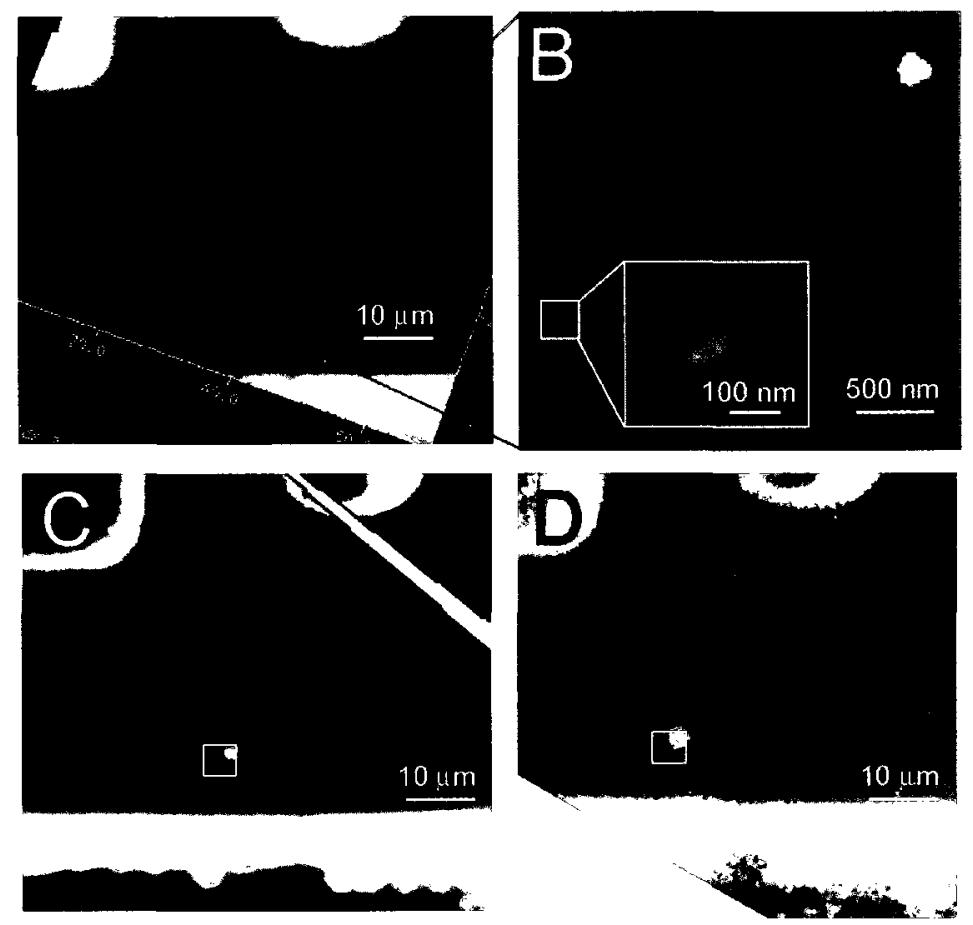

Figure 14 Individual gold nanorod $\sim 50 \times 15 \mathrm{~nm}$. The first AFM image (A) shows a region near alignment marks that are also visible in the optical images ((C) and (D)). A zoomed AFM image (B) reveals a large nanosphere in the upper right (triangular shape is a tip artifact) and a nanorod in the lower left. The nanorod is clearly revealed in the inset. Its size is exaggerated by the tip. Dark-field images captured with a high-sensitivity $\mathrm{CCD}(\mathrm{C})$ and color $\mathrm{CCD}$ (D) clearly show the nanosphere, but show no sign of the nanorod in the expected region, even with significant contrast enhancement.

In order to measure the differential scattering cross section of a single nanorod by dark field microscpectroscopy, an isolated patch of nanorods was created by scratching a nanorod film. The one micron square patch contained 28 isolated nanorods with an average size of $40 \times 13 \mathrm{~nm}$, as determined by AFM. The dark field scattering rate was analyzed as above and divided by the number of nanorods in the patch to obtain the average cross section of a single nanorod (Figure 14). The nanorod, being much smaller, is 100 times less bright than the bipyramid in the dark field microscope. The peak is broadened due to the presence of various shapes of nanorods in the patch, which were found to have lengths ranging from 33 to $57 \mathrm{~nm}$ and diameters ranging from 10 to $15 \mathrm{~nm}$. The noise in the measurement is a result of being at the lower detection limit. 


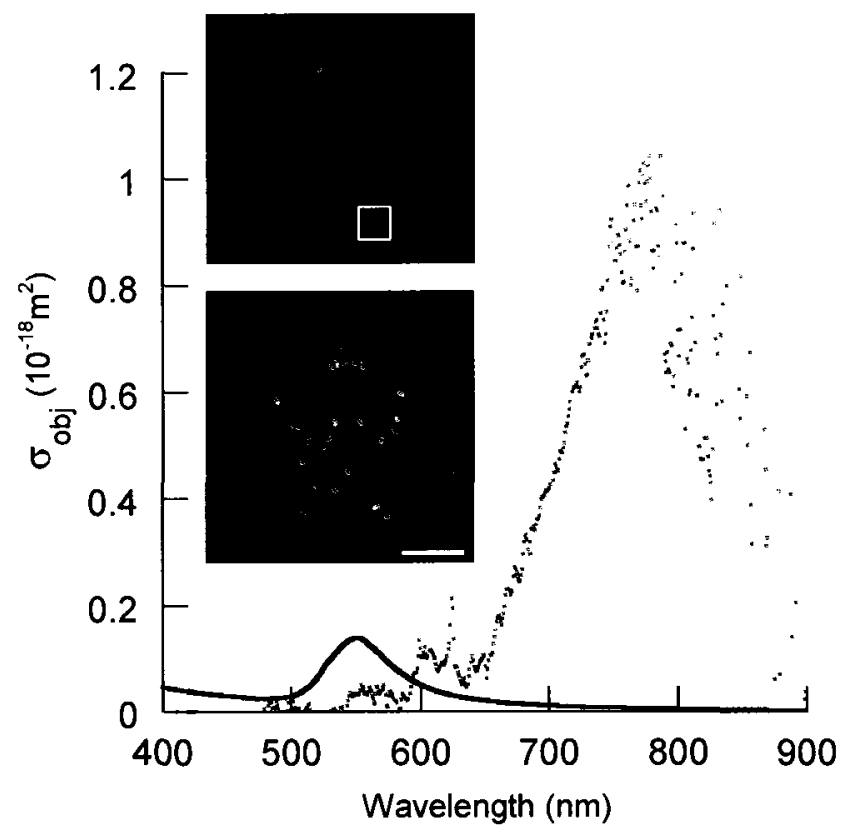

Figure 14 The average scattering cross section for a single nanorod (orange) measured from a patch of 28 nanorods shown in dark field microscopy (top inset) and AFM (bottom inset, scale bar $=500 \mathrm{~nm}$ ). The calculated equivalent gold sphere cross section is also plotted (blue).

For an ellipse of this size, the single particle approximations that are included in the Gans expression is correct to within $10 \%$. Figure 15 shows the Gans solution total scattering cross section averaged over all illumination angles and nanorod sizes from the patch in Figure 14. After fitting the average Gans solution to a Gaussian distribution, we find that the ratio between the scattering peak of the elongated particle and the spherical particle is approximately equal to the value we measured for $\sigma_{o b j}(\sim 7.5)$. Therefore, for particles of this size, the total scattering cross section is an accurate predictor of the relative contrast in dark field microscopy. For larger particles of complex shapes, however, it is likely that this relationship does not hold. Small particles tend to scatter in a dipole pattern, regardless of their shape. As particle size increases the angular scattering becomes less of a dipole pattern and exhibits more forward scattering. Particle shape will 
change this far-field scattering pattern in a way that cannot be described with simple analytical expressions.

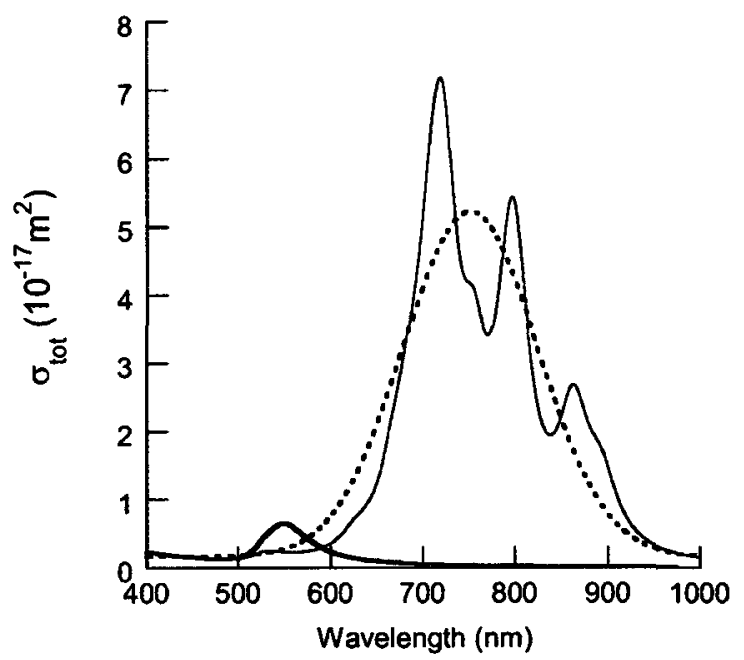

Figure 15 The average total scattering cross section calculated with the Gans Solution for the various sizes of nanorods in the spectrum measured in Figure 14 (fine line) with a Gaussian fit to that curve (dashed line) along with the total scattering cross section for a sphere of the equivalent average volume (bold line). 


\section{$\underline{\text { Discussion }}$}

Our measurements show that elongated plasmon resonant nanoparticles scatter more brightly than their equivalent volume spheres. This is due to the reduced plasmon damping in gold at the near-infrared wavelengths of their peak plasmon resonance. ${ }^{11}$ However, many other factors may affect the observed scattering signal, such as the increased wavelength, polarization dependence, and angular pattern of the scattered light. The Gans solution is only applicable to very small particles, therefore, in addition to the shape mismatch, it is of no use in predicting bipyramid scattering.

These results are in good agreement with the report by Schultz et al. ${ }^{29}$ The 109 $\mathrm{nm}$ sphere in Figure 12A, integrated over detectable wavelengths, generates approximately $10^{6}$ counts per second from the CCD, which correlates well to the photon flux reported by Schultz et al given that our measurement is on a larger nanoparticle made of gold (as opposed to silver which supports stronger plasmon resonances), and using a lower NA objective. Even bright fluorophores with extinction coefficients as high as $100,000 \mathrm{M}^{-1} \mathrm{~cm}^{-1}$ and high quantum efficiencies have emission cross sections on the order of $10^{-22} \mathrm{~m}^{2}$. Our results establish a baseline cross section detectivity of approximately $10^{-18} \mathrm{~m}^{2}$ for single nanoparticle scattering in the dark field setup described here, but that is certainly not a lower limit. Transmitted illumination dark field microscopy with both oil immersion condenser and objective generally provides more signal (due to the higher NA) and a darker background. Also, this geometry will capture scattering in the forward hemisphere, which is predominant for larger nanoparticles. 
However, this imaging mode may prove more difficult to calibrate since the illumination is manually aligned.

\section{Conclusion}

The spectral scattering for a $\mathrm{NA}=0.5$ dark field microspectrometer was calibrated using measurements on gold nanospheres and the corresponding full Mie theory solution. The calibration was applied to gold nanorods and bipyramids to quantitatively characterize their spectral scattering properties. These results show that elongated particles scatter strongly for their volume and are ideal for many applications which require small, bright nanoparticles. The brightness of small elongated nanoparticles can be accurately predicted by simple small-particle formulas for the total scattering cross section. Larger particles, however, would be best treated with numerical calculations. 


\section{$\underline{\text { References }}$}

1. Maier, S. A.; Atwater, H. A., Plasmonics: Localization and guiding of electromagnetic energy in metal/dielectric structures. Journal of Applied Physics 2005, 98, (1), -.

2. Bohren, C. F.; Huffman, D. R., Absorption and Scattering of Light by Small Particles. 2 ed.; Wiley-Interscience: 1998; p 544.

3. Anker, J. N.; Hall, W. P.; Lyandres, O.; Shah, N. C.; Zhao, J.; Van Duyne, R. P., Biosensing with plasmonic nanosensors. Nature Materials 2008, 7, (6), 442-453.

4. Murphy, C. J.; Gole, A. M.; Stone, J. W.; Sisco, P. N.; Alkilany, A. M.; Goldsmith, E. C.; Baxter, S. C., Gold Nanoparticles in Biology: Beyond Toxicity to Cellular Imaging. Accounts of Chemical Research 2008, 41, (12), 1721-1730.

5. Liao, H.; Nehl, C. L.; Hafner, J. H., Biomedical applications of plasmon resonant metal nanoparticles. Nanomedicine 2006, 1, (2), 201-208.

6. Lal, S.; Link, S.; Halas, N. J., Nano-optics from sensing to waveguiding. Nature Photonics 2007, 1, (11), 641-648.

7. Link, S.; Mohamed, M.; El-Sayed, M., Simulation of the optical absorption spectra of gold nanorods as a function of their aspect ratio and the effect of the medium dielectric constant. Journal of Physical Chemistry B 1999, 103, (16), 3073-3077.

8. $\quad$ Klar, T.; Perner, M.; Grosse, S.; von Plessen, G.; Spirkl, W.; Feldmann, J., Surface-plasmon resonances in single metallic nanoparticles. Physical Review Letters 1998, 80, (19), 4249-4252.

9. $\quad$ Sonnichsen, C.; Geier, S.; Hecker, N. E.; von Plessen, G.; Feldmann, J.; Ditlbacher, H.; Lamprecht, B.; Krenn, J. R.; Aussenegg, F. R.; Chan, V. Z. H.; Spatz, J. P.; Moller, M., Spectroscopy of single metallic nanoparticles using total internal reflection microscopy. Applied Physics Letters 2000, 77, (19), 2949-2951.

10. Mock, J. J.; Barbic, M.; Smith, D. R.; Schultz, D. A.; Schultz, S., Shape effects in plasmon resonance of individual colloidal silver nanoparticles. Journal of Chemical Physics 2002, 116, (15), 6755-6759.

11. Sönnichsen, C.; Franzl, T.; Wilk, T.; von Plessen, G.; Feldmann, J., Drastic Reduction of Plasmon Damping in Gold Nanorods. Physical Review Letters 2002, 88, (7), 077402.

12. Mock, J. J.; Smith, D. R.; Schultz, S., Local refractive index dependence of plasmon resonance spectra from individual nanoparticles. Nano Letters 2003, 3, (4), 485491.

13. Raschke, G.; Kowarik, S.; Franzl, T.; Sonnichsen, C.; Klar, T. A.; Feldmann, J.; Nichtl, A.; Kurzinger, K., Biomolecular recognition based on single gold nanoparticle light scattering. Nano Letters 2003, 3, (7), 935-938.

14. McFarland, A. D.; Van Duyne, R. P., Single Silver Nanoparticles as Real-Time Optical Sensors with Zeptomole Sensitivity. Nano Letters 2003, 3, 1057-1062.

15. Nehl, C.; Grady, N.; Goodrich, G.; Tam, F.; Halas, N.; Hafner, J., Scattering spectra of single gold nanoshells. Nano Letters 2004, 4, (12), 2355-2359. 
16. Sherry, L. J.; Chang, S.-H.; Schatz, G. C.; van Duyne, R. P., Localized Surface Plasmon Resonance Spectroscopy of Single Silver Nanocubes. Nano Letters 2005, 5 , (10), 2034-2038.

17. Nehl, C. L.; Liao, H. W.; Hafner, J. H., Optical properties of star-shaped gold nanoparticles. Nano Letters 2006, 6, (4), 683-688.

18. Liu, M.; Guyot-Sionnest, P., Mechanism of Silver(I)-Assisted Growth of Gold Nanorods and Bipyramids. Journal of Physical Chemistry B 2005, 109, (47), 2219222200.

19. Markel, V. A.; Shalaev, V. M.; Zhang, P.; Huynh, W.; Tay, L.; Haslett, T. L.; Moskovits, M., Near-field optical spectroscopy of individual surface-plasmon modes in colloid clusters. Physical Review B 1999, 59, (16), 10903-10909.

20. Dorfmuller, J.; Vogelgesang, R.; Weitz, R. T.; Rockstuhl, C.; Etrich, C.; Pertsch, T.; Lederer, F.; Kern, K., Fabry-Perot Resonances in One-Dimensional Plasmonic Nanostructures. Nano Letters 2009, 9, (6), 2372-2377.

21. Schaffer, B.; Hohenester, U.; Trugler, A.; Hofer, F., High-resolution surface plasmon imaging of gold nanoparticles by energy-filtered transmission electron microscopy. Physical Review B 2009, 79, (4), -.

22. Eggeman, A. S.; Dobson, P. J.; Petford-Long, A. K., Optical spectroscopy and energy-filtered transmission electron microscopy of surface plasmons in core-shell nanoparticles. Journal of Applied Physics 2007, 101, (2), -.

23. Gomez-Medina, R.; Yamamoto, N.; Nakano, M.; Abajo, F. J. G., Mapping plasmons in nanoantennas via cathodoluminescence. New Journal of Physics 2008, 10, -. 24. Van Wijngaarden, J. T.; Verhagen, E.; Polman, A.; Ross, C. E.; Lezec, H. J.; Atwater, H. A., Direct imaging of propagation and damping of near-resonance surface plasmon polaritons using cathodoluminescence spectroscopy. Applied Physics Letters 2006, 88, (22), -.

25. Liao, H.; Hafner, J., Gold nanorod bioconjugates. Chemistry of Materials 2005, $17,(18), 4636-4641$.

26. Orendorff, C. J.; Murphy, C. J., Quantitation of Metal Content in the SilverAssisted Growth of Gold Nanorods. Journal of Physical Chemistry B 2006, ASAP.

27. Chen, Y.; Munechika, K.; Plante, I. J. L.; Munro, A. M.; Skrabalak, S. E.; Xia, Y.; Ginger, D. S., Excitation enhancement of CdSe quantum dots by single metal nanoparticles. Applied Physics Letters 2008, 93, (5), -.

28. Chen, Y.; Munechika, K.; Ginger, D. S., Dependence of fluorescence intensity on the spectral overlap between fluorophores and plasmon resonant single silver nanoparticles. Nano Letters 2007, 7, (3), 690-696.

29. Schultz, S.; Smith, D. R.; Mock, J. J.; Schultz, D. A., Single-target molecule detection with nonbleaching multicolor optical immunolabels. Proceedings of the National Academy of Sciences of the United States of America 2000, 97, (3), 996-1001. 30. Encina, E. R.; Coronado, E. A., Plasmonic nanoantennas: Angular scattering properties of multipole resonances in noble metal nanorods. Journal of Physical Chemistry C 2008, 112, (26), 9586-9594.

31. Jain, P. K.; Lee, K. S.; El-Sayed, I. H.; El-Sayed, M. A., Calculated absorption and scattering properties of gold nanoparticles of different size, shape, and composition: Applications in biological imaging and biomedicine. Journal of Physical Chemistry B 2006, 110, (14), 7238-7248. 
32. Ni, W.; Kou, X.; Yang, Z.; Wang, J. F., Tailoring longitudinal surface plasmon wavelengths, scattering and absorption cross sections of gold nanorods. Acs Nano 2008, 2, (4), 677-686.

33. Papavissiou, G. C., Optical Properties of Small Inorganic qand Organic Metal Particles. Progess in Solid State Chemistry 1979, 12, (3-4), 185-271.

34. Asano, S.; Yamamoto, G., Light-Scattering by a Spheroidal Particle. Applied Optics 1975, 14, (1), 29-49.

35. Qiu, L.; Larson, T. A.; Smith, D. K.; Vitkin, E.; Zhang, S. H.; Modell, M. D.; Itzkan, I.; Hanlon, E. B.; Korgel, B. A.; Sokolov, K. V.; Perelman, L. T., Single gold nanorod detection using confocal light absorption and scattering spectroscopy. Ieee Journal of Selected Topics in Quantum Electronics 2007, 13, (6), 1730-1738.

36. Bryant, G. W.; De Abajo, F. J. G.; Aizpurua, J., Mapping the plasmon resonances of metallic nanoantennas. Nano Letters 2008, 8, (2), 631-636.

37. Knight, M. W.; Wu, Y. P.; Lassiter, J. B.; Nordlander, P.; Halas, N. J., Substrates Matter: Influence of an Adjacent Dielectric on an Individual Plasmonic Nanoparticle. Nano Letters 2009, 9, (5), 2188-2192.

38. Rostro, B. C.; Bickford, L.; Hafner, J. H., Stabilization and Targeting of Surfactant-Synthesized Gold Nanorods. Nanotechnology 2009, In press.

39. Zuchner, T.; Wackenhut, F.; Failla, A. V.; Meixner, A. J., Nanoscale characterization of single Au nanorods by confocal microscopy. Applied Surface Science 2009, 255, (10), 5391-5395.

40. Javier, D. J.; Nitin, N.; Roblyer, D. M.; Richards-Kortum, R., Metal-based nanorods as molecule-specific contrast agents for reflectance imaging in 3D tissues.

Journal of Nanophotonics 2008, 2, -. 


\section{$\underline{\text { Appendix }}$}

\section{Appendix A}

\section{Mie Solution: Calculating $\sigma_{\text {tot }}$ and $\sigma_{0 b j}$}

clear

\%input nanosphere radius

din=input('diameter in nm? ','s');

$\%$ radius $=$ diameter $/ 2$

$\operatorname{rad}=\operatorname{str} 2$ num $(\operatorname{din}) / 2$;

$r=r^{*}$ le-9;

load gold diel2

$\mathrm{nm}=1.515$;

$\mathrm{n}=$ gold_dielectric $2(:, 2)$;

$\mathrm{k}=$ gold_dielectric $2(:, 3)$;

$\%$ input angular detection range

$\%$ minimum detection angle (radians)

thetaMin=3.2909;

\%maximum detection angle (radians)

thetaMax=3.9635;

$\%$ phi range $=$ theta range

phi=thetaMax-thetaMin;

for $\mathrm{t}=1: 600$;

$w(t)=t+400 ;$

$\mathrm{m}=\left(\mathrm{n}(\mathrm{t})+\mathrm{i}^{*} \mathrm{k}(\mathrm{t})\right) / \mathrm{nm}$; \%ratio of indecies of refraction particle/medium

$\mathrm{l}=\mathrm{t}+400 ; \%$ wavelegth $(\mathrm{nm})$

$\mathrm{kn}=\left(2^{*} \mathrm{pi}^{*} \mathrm{~nm}\right) /\left(1^{*} 10^{\wedge}(-9)\right) ; \%$ wavenumber

$\mathrm{x}=\mathrm{kn} * \mathrm{r}$;

\%getting "a" and "b" ( $\mathrm{L}$ is the multipole order)

for $\mathrm{L}=1: 9$

$\operatorname{psix}=\operatorname{sqrt}\left(\left(\mathrm{pi}^{*} \mathrm{x}\right) / 2\right)^{*} \operatorname{besselj}(\mathrm{L}+(1 / 2), \mathrm{x})$;

$\operatorname{psix} 2=\operatorname{sqrt}(\mathrm{pi} / 2)^{*}\left(((\mathrm{~L}+1) / \operatorname{sqrt}(\mathrm{x}))^{*} \operatorname{besselj}(\mathrm{L}+(1 / 2), \mathrm{x})-\right.$

$\left(\operatorname{sqrt}(\mathrm{x})^{*}\right.$ besselj $\left.\left.(\mathrm{L}+(3 / 2), \mathrm{x})\right)\right)$;

$\operatorname{psim} x=\operatorname{sqrt}\left(\mathrm{pi}^{*} \mathrm{~m}^{*} \mathrm{x} / 2\right)^{*}$ besselj $\left(\mathrm{L}+(1 / 2), \mathrm{m}^{*} \mathrm{x}\right)$;

$\operatorname{psim} x 2=\operatorname{sqrt}(\mathrm{pi} / 2) *\left(\left((\mathrm{~L}+1) / \operatorname{sqrt}\left(\mathrm{m}^{*} \mathrm{x}\right)\right)^{*} \operatorname{besselj}\left(\mathrm{L}+(1 / 2), \mathrm{m}^{*} \mathrm{x}\right)-\right.$

$\left(\operatorname{sqrt}\left(\mathrm{m}^{*} \mathrm{x}\right) *\right.$ besselj$\left.\left.\left(\mathrm{L}+(3 / 2), \mathrm{m}^{*} \mathrm{x}\right)\right)\right)$;

$\mathrm{ksix}=\operatorname{sqrt}\left(\mathrm{pi}^{*} \mathrm{x} / 2\right) *$ besselh $(\mathrm{L}+(1 / 2), \mathrm{x})$;

$\operatorname{ksix} 2=\operatorname{sqrt}(\mathrm{pi} / 2) *((\mathrm{~L}+1) / \operatorname{sqrt}(\mathrm{x}))^{*} \operatorname{besselh}(\mathrm{L}+(1 / 2), \mathrm{x})-$

$\left.\left(\operatorname{sqrt}(\mathrm{x})^{*} \operatorname{besselh}(\mathrm{L}+(3 / 2), \mathrm{x})\right)\right)$;

$\mathrm{ksim} x=\operatorname{sqrt}\left(\mathrm{pi}^{*} \mathrm{~m}^{*} \mathrm{x} / 2\right)^{*}$ besselh $\left(\mathrm{L}+(1 / 2), \mathrm{m}^{*} \mathrm{x}\right)$;

$\operatorname{ksim} x 2=\operatorname{sqrt}(\mathrm{pi} / 2) *\left(\left((\mathrm{~L}+1) / \operatorname{sqrt}\left(\mathrm{m}^{*} \mathrm{x}\right)\right)^{*}\right.$ besselh $\left(\mathrm{L}+(1 / 2), \mathrm{m}^{*} \mathrm{x}\right)-$

$\left(\operatorname{sqrt}\left(m^{*} x\right)^{*}\right.$ besselh $\left.\left.\left(\mathrm{L}+(3 / 2), \mathrm{m}^{*} \mathrm{x}\right)\right)\right)$;

$\mathrm{a}(\mathrm{L})=\left(\mathrm{m}^{*} \mathrm{p} \operatorname{sim} \mathrm{x}^{*} \mathrm{psix} 2-\mathrm{psix} * \mathrm{p} \operatorname{sim} \mathrm{x} 2\right) /\left(\mathrm{m}^{*} \mathrm{p} \operatorname{sim} \mathrm{x}^{*} \mathrm{ksix} 2-\mathrm{ksix} * \mathrm{psim} \mathrm{x} 2\right)$;

$b(L)=\left(p \operatorname{sim} x^{*} p \operatorname{six} 2-m^{*} p \operatorname{six}{ }^{*} p \operatorname{sim} x 2\right) /\left(p \operatorname{sim} x^{*} k \operatorname{six} 2-m^{*} k^{*} \operatorname{six}{ }^{*} p \operatorname{sim} x 2\right)$; 


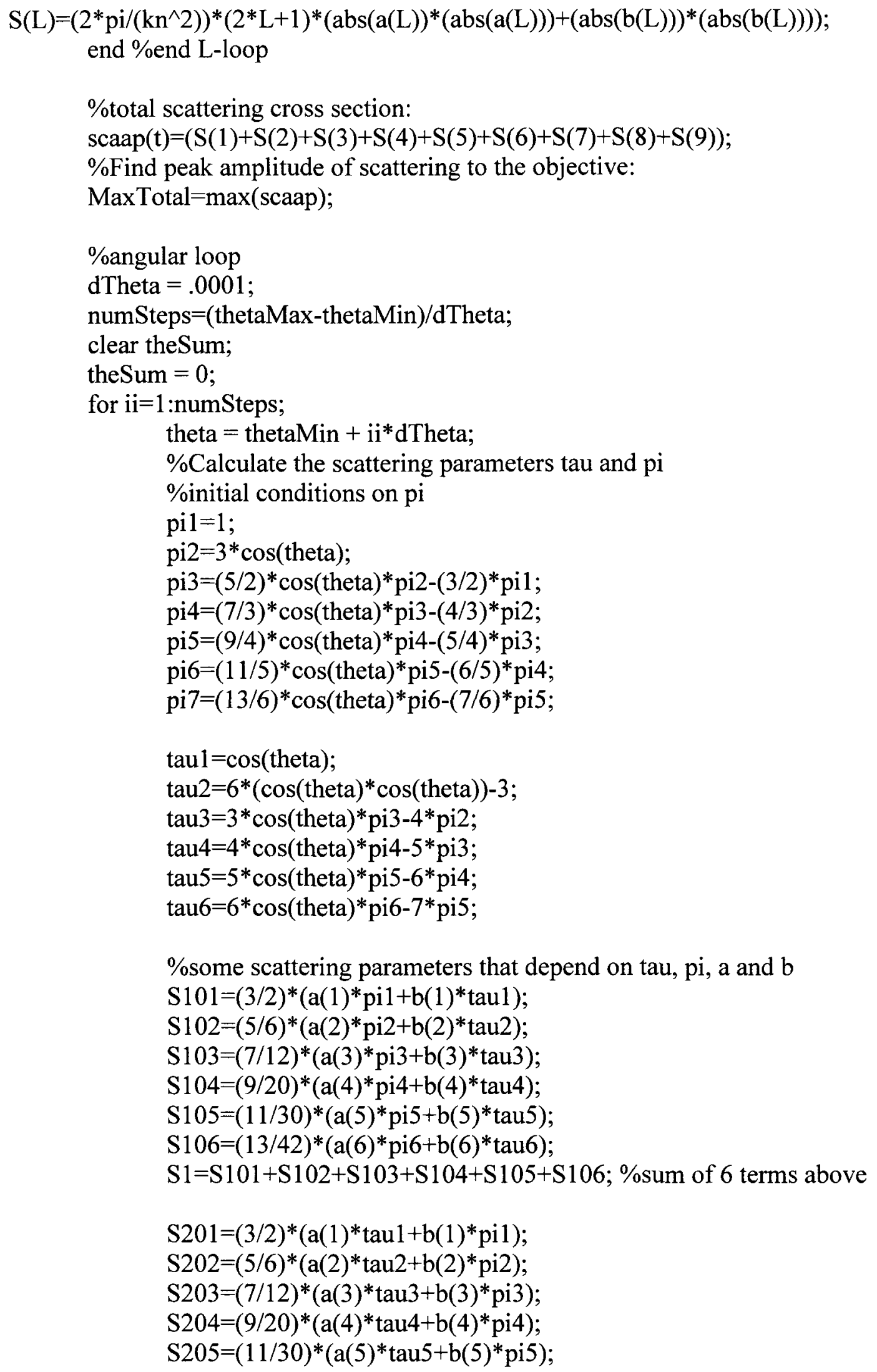


$\mathrm{S} 206=(13 / 42) *(\mathrm{a}(6) * \operatorname{tau} 6+\mathrm{b}(6) * \mathrm{pi} 6)$

$\mathrm{S} 2=\mathrm{S} 201+\mathrm{S} 202+\mathrm{S} 203+\mathrm{S} 204+\mathrm{S} 205+\mathrm{S} 206 ; \%$ sum of 6 terms above

$\mathrm{S} 11=.5 *\left(\operatorname{abs}(\mathrm{S} 2)^{*} \mathrm{abs}(\mathrm{S} 2)+\mathrm{abs}(\mathrm{S} 1)^{*} \mathrm{abs}(\mathrm{S} 1)\right)$;

ds_dt $=\mathrm{S} 11 /(\mathrm{kn} * \mathrm{kn})$;

theSum $=$ theSum + abs $\left(d s \_d t * \sin (\right.$ theta $\left.)\right) *$ dTheta;

end

$\%$ Scattering cross section for wavelength $t$ over the solid angle of the \%detector:

scatter $(\mathrm{t})=$ phi*theSum;

end

\%Find peak amplitude of scattering to the objective:

MaxDiff=max(scatter);

\%Find peak wavelength:

temporary=scatter;

for ii $=1: 600$

if MaxDiff==temporary(ii)

peakpsc $=\mathrm{ii}$;

end

end

peak_wavelength_sc $=$ peakpsc +400 ; 


\section{Appendix B}

\section{Comparing $\sigma_{\text {tot }}$ (dipole term only, small particle approximation, Gans solution)}

clear

$\%$ Calculate the extinction, absorption, and scattering from a gold sphere or \%ellipsoid using Mie or Gans theory (Gan's is in the small particle limit).

load gold_diel

$\mathrm{n} 2=$ input('Medium index of refraction (n)?', 's');

$\mathrm{nm}=\operatorname{str} 2$ num(n2);

$\mathrm{em}=\mathrm{nm}^{\wedge} 2$;

type=input('Would you like to model a sphere or an ellipsoid? (sphere $=0$, ellipsoid=1):','s');

model=str2num(type);

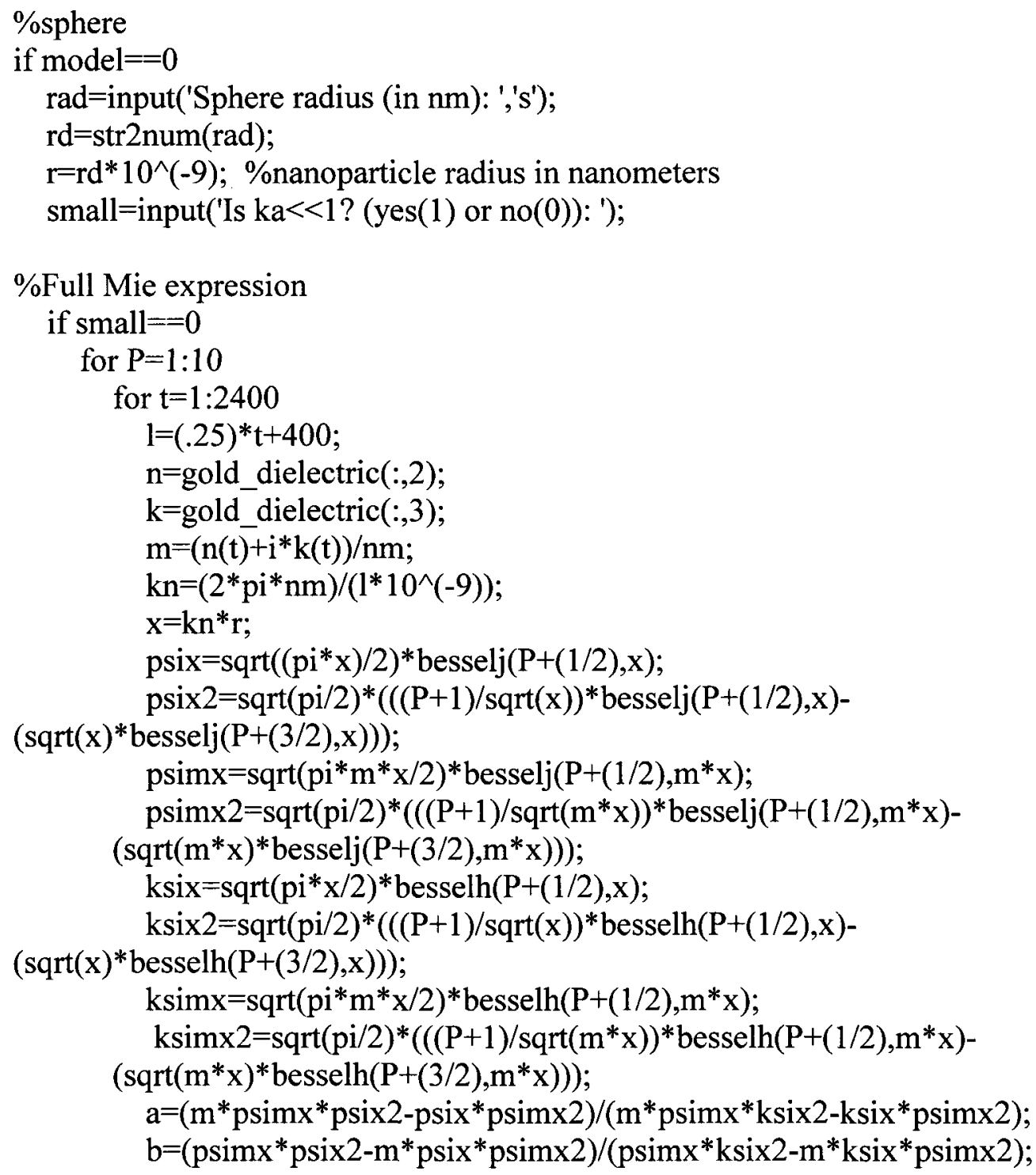




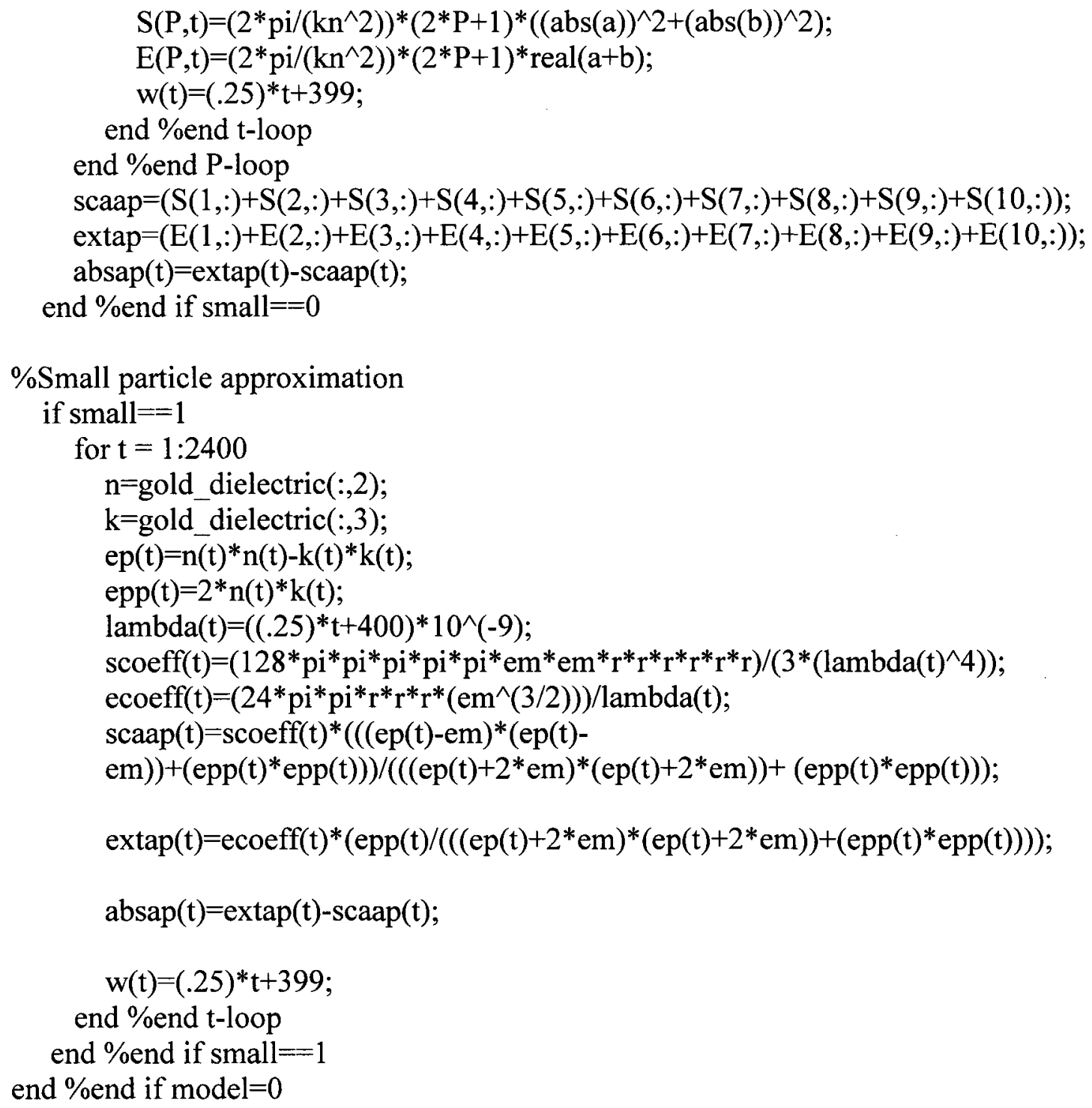




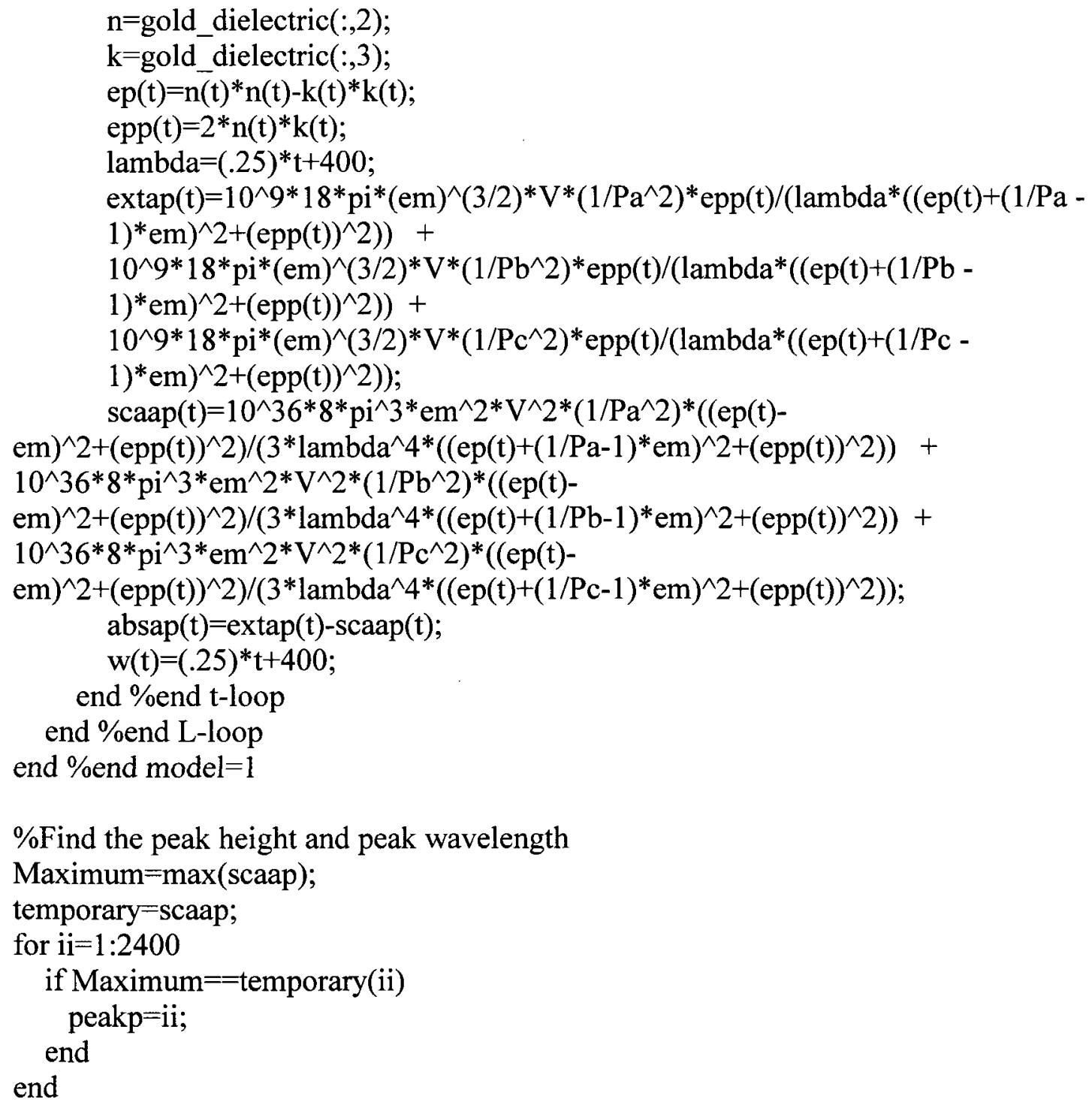




\section{Appendix C \\ Analyzing Data and Calculating $K_{\lambda}$}

clear

load Mie; \%load angular mie result

load std; \%load spectral wavelength values

$\%$ Create wavelength values $400-1000 \mathrm{~nm}$

for $\mathrm{t}=1: 600$

$\mathrm{w}(\mathrm{t})=\mathrm{t}+400$;

end

\%Analyze spectral data:

$\%$ Find the file and save the filename and path

[file,path]=uigetfile('*','Select the image to analyze');

\%Load the image

ve $=$ imread([path,file],'tiff $)$;

$\%$ convert integers to floating point numbers

$\mathrm{ve}=$ double(ve);

$\%$ flip the image upside down so orientation appears same as original

for $\mathrm{i}=1: 512$

for $\mathrm{j}=1: 512$ $\operatorname{vd}(\mathrm{i}, \mathrm{j})=\operatorname{ve}(513-\mathrm{i}, \mathrm{j})$;

end

end

$\%$ Plot the image to specify particle location and width

figure

drawnow

pcolor(vd)

shading interp

st = input('Based on the image, enter the pixel of the spectrum center: ','s');

center $=\operatorname{str} 2$ num(st);

stl = input('What width (in pixels) do you want to sum over? ','s');

width $=\operatorname{str} 2$ num $($ st1 $)$;

$\mathrm{dt}=$ input('What is the integration time of the image? ','s');

time $=$ str2num(dt);

$\%$ isolate data in this range (where the particle is) 


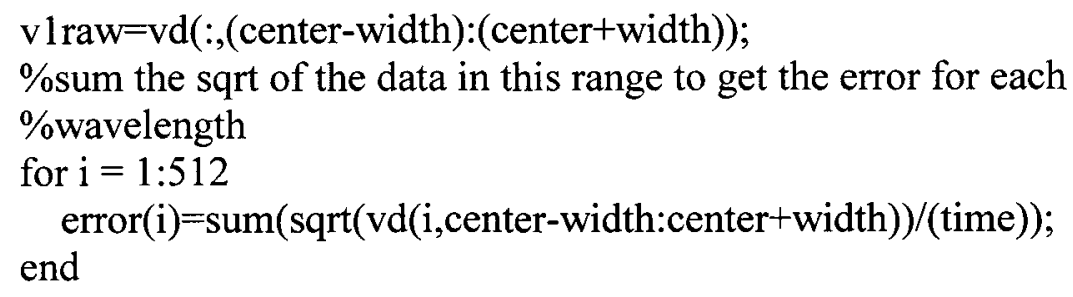

$\%$ create $\mathrm{x}$-axis: spatial values

$\mathrm{x}=1: 512$;

$\mathrm{x}(($ center-width $):($ center + width $))=[]$;

\%subtract particle out of data (for bkg fit)

$\operatorname{vd}(:,($ center-width $):($ center+width $))=[]$;

$\operatorname{plot}(\mathrm{x}, \mathrm{vd}(:,:))$

$\%$ choose range to fit background (in pixel values)

$\mathrm{rl}=$ input('Lower bound on background fit? ','s');

range $1=\operatorname{str} 2$ num $(\mathrm{rl})$;

$\mathrm{ru}=$ input('Upper bound on background fit? ','s');

range $2=\operatorname{str} 2$ num(ru);

for $\mathrm{ii}=1:\left(512-\left(\left(2^{*}\right.\right.\right.$ width $\left.\left.)+1\right)\right)$

if $x(\mathrm{ii})==$ range 1

$\mathrm{rl}=\mathrm{ii}$;

end

if $\mathrm{x}(\mathrm{ii})==$ range 2

r2=ii;

end

end \%end ii-loop

$\mathrm{vd}=\mathrm{vd}(:,(\mathrm{r} 1+1):(\mathrm{r} 2-1))$;

$\%$ Take out edges and change the $\mathrm{x}$-axis accordingly

$\mathrm{x}(\mathrm{r} 2:(512-((2 *$ width $)+1)))=[]$;

$\mathrm{x}(1: \mathrm{r} 1)=[]$;

$\mathrm{N}=$ input('What order polynomial would you like to fit the background to?' );

for $\mathrm{i}=1: 512 \%$ (each wavelength)

drawnow

$\operatorname{plot}(\mathrm{x}, \mathrm{vd}(\mathrm{i}, \mathrm{:}))$

hold on

$\%$ for each wavelength, fit a curve to vd(signal-paricle) as a function of

$\%$ space

$\mathrm{P}=\operatorname{polyfit}(\mathrm{x}, \mathrm{vd}(\mathrm{i},:), \mathrm{N})$;

for $\mathrm{ii}=1:(\mathrm{r} 2-1-\mathrm{r} 1) \%$ each spatial value

$\%$ Nth order

for $\mathrm{j}=1: \mathrm{N}+1$

$v b 2(j)=P(j)^{*} x(i i)^{\wedge}(N-(j-1))$; 


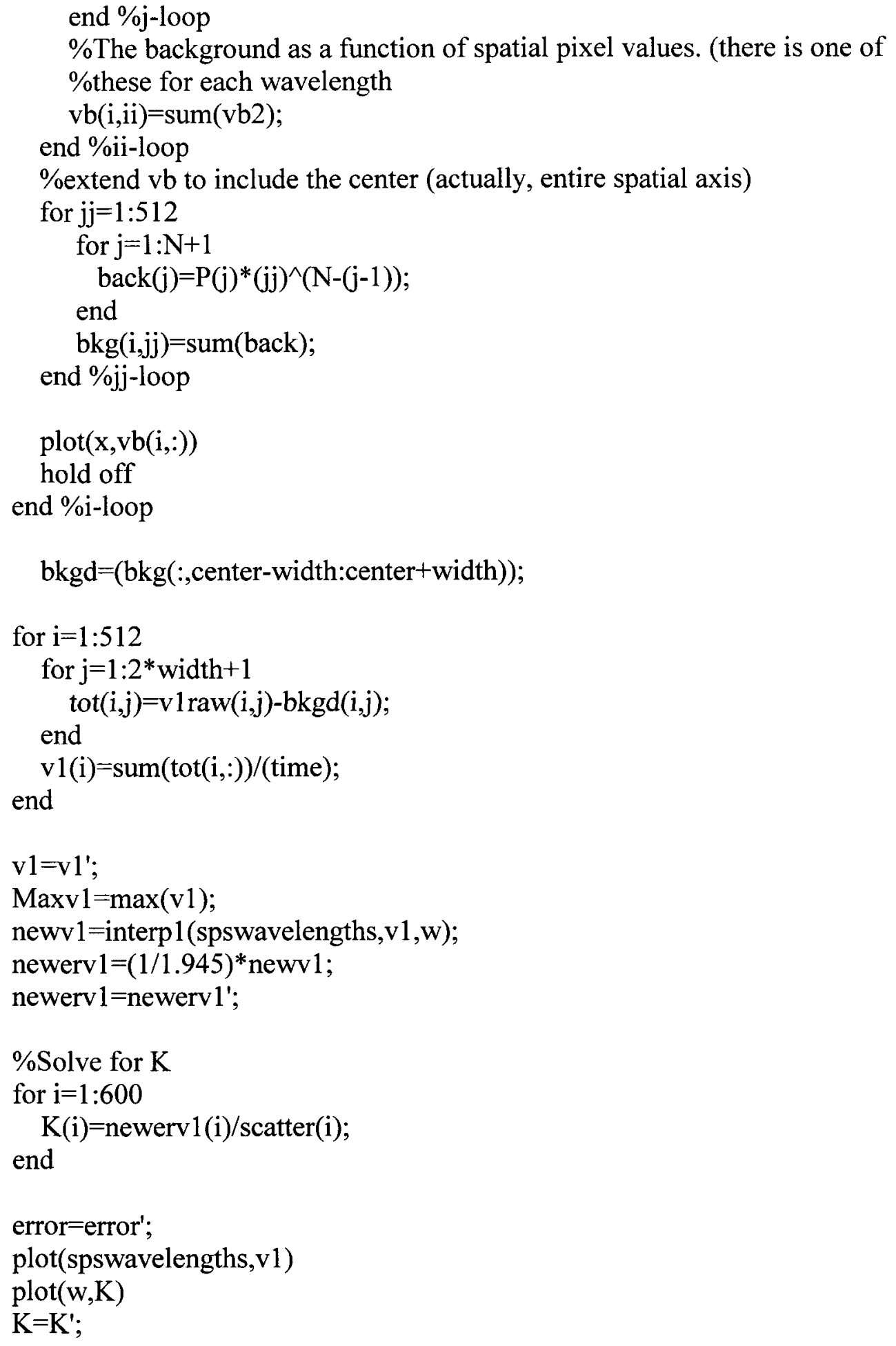

